\title{
Thermospheric responses to gravity waves arising from mesoscale convective complexes
}

\author{
Sharon L. Vadas*, David C. Fritts \\ Colorado Research Associates, A Division of NorthWest Research Associates, 3380 Mitchell Lane, Boulder, CO 80301, USA
}

Received 24 March 2003; accepted 20 January 2004

\begin{abstract}
We employ a linear model of the responses to local body forces to estimate the spectra of gravity waves arising due to vertical motions within mesoscale convective complexes (MCCs) at equatorial latitudes. Ray tracing methods are then used to propagate these spectra through model wind and temperature fields and to anticipate their thermospheric effects. We find that gravity wave forcing by MCCs is dominated by large vertical motions of limited horizontal extent, that individual convective cells within an MCC effectively act as independent sources of gravity waves if they are separated by two or more diameters, and that vertical body forces create higher-frequency gravity waves than comparable horizontal body forces. Ray tracing reveals that of the gravity waves excited by MCCs, only the high-frequency, long-vertical-wavelength portions of the refracted spectrum (above the shear) can penetrate to high altitudes, that variable winds impose significant anisotropy on the surviving gravity waves, that refraction and dissipation determine the portion of the MCC wave spectrum important in the lower thermosphere, and that large net momentum flux divergence may lead to strong local body forcing well into the thermosphere. Gravity waves excited by MCCs and having sufficiently large vertical wavelengths and group velocities above the shears at lower altitudes can penetrate to altitudes at which they may be expected to contribute to the seeding of equatorial spread $\mathrm{F}$. (c) 2004 Elsevier Ltd. All rights reserved.
\end{abstract}

Keywords: Gravity waves; Thermosphere dynamics; Mesoscale convective complexes

\section{Introduction}

Numerous studies have addressed the excitation and effects of gravity waves arising from strong convection as their roles in the stratosphere and at greater altitudes have become increasingly recognized. Observational studies have associated gravity waves near the mesopause with severe storms (Taylor and Hapgood, 1988; Sentman et al., 2003) or correlated mesoscale variance, discrete gravity wave signatures, and wave momentum fluxes in the troposphere and lower stratosphere with convection and frontal activity (Larsen et al., 1982; Lu et al., 1984; Kuettner et al., 1987; Fritts and Nastrom, 1992; Hauf, 1993; Pfister et al., 1993a, b; Alexander and Pfister, 1995; Sato et al., 1995; Karoly et al.,

\footnotetext{
* Corresponding author. Tel.: +1-303-415-9701x202; fax: +1-303-415-9702.

E-mail address: vasha@cora.nwra.com (S.L. Vadas).
}

1996; Dewan et al., 1998; Alexander et al., 2000; Dhaka et al., 2001, 2002). The modeling study by Alexander and Vincent (2000) concluded that the seasonal and interannual variability of gravity waves observed at Cocos Island and described by Vincent and Alexander (2000) was likely due to deep convection. Other studies estimated that convective gravity waves make a significant contribution to mean forcing (Dunkerton, 1997; Alexander and Holton, 1997; Chun and Baik, 1998; Piani et al., 2000).

More focused studies of convection and gravity wave generation examined the specific mechanisms of wave generation. Two-dimensional (2D) studies suggested three primary sources of gravity waves arising from convection. These are the "mechanical oscillator" (Clark et al., 1986; Fovell et al., 1992; Alexander et al., 1995), deep heating (Salby and Garcia, 1987; Walterscheid et al., 2001; Holton et al., 2002), and the "obstacle effect" (Pfister et al., 1993a; Alexander and Vincent, 2000), which appear to play varying roles depending on the spatial and temporal structure of the 
convection and the environmental shear and stability profiles. More recent studies have emphasized (or reiterated) the three-dimensional (3D) aspects of convectively generated gravity waves and further quantified their structure, source mechanisms, and responses to environmental profiles (Piani et al., 2000; Lane et al., 2001; Horinouchi et al., 2002; Beres et al., 2002, 2004). In particular, these studies have highlighted the tendency for the resulting wave field to be determined largely by the responses to local, rather than "ensemble", forcing by apparently individual convective cells, with the circular patterns of gravity waves radiating outward from the most intense convection often seen well above the convective sources (Taylor and Hapgood, 1988; Dewan et al., 1998; Sentman et al., 2003). Further discussion of convectively generated gravity waves, and of their effects at higher altitudes, may be found in the recent review by Fritts and Alexander (2003).

The gravity waves excited by convection have a potential to have influences to very high altitudes because they often have relatively large vertical wavelengths and they often propagate, initially at least, in all directions from the convective source. Large vertical wavelengths imply large horizontal phase speeds that escape critical-level absorption more readily than waves having smaller phase speeds. Quasi-isotropic directional propagation implies that at least a portion of the excited waves will penetrate to high altitudes, where increasing large-scale winds cause waves to refract to higher and lower intrinsic frequencies (and phase speeds) and allow for differential momentum deposition where the waves are dissipated (Vadas and Fritts, 2002). The largest vertical wavelengths (and phase speeds) also are favored by deep convection (Salby and Garcia, 1987), and the most energetic waves are excited by the most intense convection having the strongest heating and the largest vertical motions (Piani et al., 2000; Lane et al., 2001). The combination of these influences makes the tropical convection zones the dominant sources of convectively generated gravity waves, and possibly the dominant sources of high-frequency gravity waves on the planet, based on wave variances inferred from GPS and other data (Tsuda et al., 2000).

The consequences of gravity waves penetrating to very high altitudes are largely unknown at this time. However, there is some evidence that they propagate to altitudes of $\sim 200-400 \mathrm{~km}$ and above, and that they may have some significant effects on the neutral and plasma dynamics at these altitudes. Electron density and temperature measurements with VHF and UHF radars suggest gravity wave fluctuations having periods of $\sim 40 \mathrm{~min}$ to $2 \mathrm{~h}$ and vertical scales $\sim 30-50 \mathrm{~km}$ to altitudes of $\sim 100-200 \mathrm{~km}$, with larger vertical scales at greater altitudes (Hocke and Schlegel, 1996; Oliver et al., 1997). We note, however, that the evidence of gravity waves at very high altitudes (above $\sim 200 \mathrm{~km}$ ) is often linked to apparent auroral sources at high latitudes. Other measurements have suggested a possible link between stratospheric gravity waves above tropical convection with ionospheric irregularities at much greater altitudes (Hocke and Tsuda, 2001). Gravity waves have also been implicated in the seeding of equatorial spread F (ESF) (Hysell et al., 1990; Huang and Kelley, 1996a, b; Sekar and Kelley, 1998) and in large local body forcing of the lower thermosphere (Vadas and Fritts, 2002), though these effects remain to be confirmed and quantified with further modeling and observations. There are, nevertheless, sufficient motivations at this point in time to explore more fully the potential links between convectively generated gravity waves at tropical latitudes and their effects in the lower thermosphere.

Our objective in this paper is to approximate both the spectrum of gravity waves arising from mesoscale convective complexes (MCCs) at tropical latitudes and their propagation through variable wind and stability profiles into the lower thermosphere. For this purpose, we will represent the convective sources of gravity waves accompanying MCCs as momentum sources in an idealized linear model based on the formalism advanced by Vadas and Fritts (2001, 2002). Such an approach is validated by the relatively good agreement found between such idealized linear forcing and a direct nonlinear description of convectively generated gravity waves by Pandya and Alexander (1999). The resulting gravity wave spectrum is then propagated to higher altitudes via ray-tracing techniques (Eckermann, 1992; Marks and Eckermann, 1995; Eckermann and Marks, 1996), from which we infer penetration altitudes and possible wave influences.

Our paper is structured as follows. The formulation of the linear model employed to describe gravity wave excitation by MCCs and the ray-tracing methodology used to describe their vertical propagation are summarized in Section 2. Section 3 describes our model of tropical convection, and Section 4 describes the spectrum of gravity waves arising from MCCs for the assumed spatial and temporal scales of this source. Their vertical propagation through two canonical winds and an isothermal temperature profile is examined in Section 5. The possible roles of gravity waves arising from MCCs at lower altitudes for body forcing, dependence on non-isothermal thermospheric temperature profiles, and ESF seeding are also explored in Section 5. A summary and our conclusions are provided in Section 6.

\section{Model formulations}

\subsection{Body forcing model}

Convective sources of gravity waves can be described equivalently as heating or momentum sources, as these sources are coupled through the vertical momentum equation. For our purposes here, we will describe MCC convective sources as vertical body forces having spatial and temporal scales representative of the vertical motions within MCC convection. Our formulation is based on that employed previously by Vadas and Fritts $(2001,2002)$ and Vadas et al. (2003) to describe the mean and gravity wave 
responses to horizontal body forces accompanying localized gravity wave breaking and momentum deposition. Those applications employed an $f$-plane representation of the governing equations. Our current application would be best served by a $\beta$-plane representation of these same equations appropriate for equatorial latitudes. However, this would render the equations nonlinear in the latitudinal coordinate and not analytically tractable. Instead, and motivated by the expected dominance of the wave spectrum by wave motions at high intrinsic frequencies, we employ the $f$-plane formalism by Vadas and Fritts (2001) with $f=0$.

\subsubsection{Formulation}

Vadas and Fritts (2001) (hereafter VF) considered the problem of body forcings and heatings that are spatially and temporally localized. The 3D linear, Boussinesq, incompressible, $f$-plane equations assuming no background wind are

$$
\begin{aligned}
& \frac{\partial u}{\partial t}+\frac{1}{\bar{\rho}} \frac{\partial p^{\prime}}{\partial x}-f v=F_{x}(\mathbf{x}) \mathscr{F}(t), \\
& \frac{\partial v}{\partial t}+\frac{1}{\bar{\rho}} \frac{\partial p^{\prime}}{\partial y}+f u=F_{y}(\mathbf{x}) \mathscr{F}(t), \\
& \frac{\partial w}{\partial t}+\frac{1}{\bar{\rho}} \frac{\partial p^{\prime}}{\partial z}-\frac{g}{\bar{\theta}} \theta^{\prime}=F_{z}(\mathbf{x}) \mathscr{F}(t), \\
& \frac{\partial \theta^{\prime}}{\partial t}+\frac{\bar{\theta} N^{2}}{g} w=\frac{\bar{\theta}}{g} J(\mathbf{x}) \mathscr{F}(t), \\
& \frac{\partial u}{\partial x}+\frac{\partial v}{\partial y}+\frac{\partial w}{\partial z}=0
\end{aligned}
$$

where $N$ is the buoyancy frequency, $u, v$, and $w$ are the zonal, meridional, and vertical velocities, $\theta=T\left(p_{\mathrm{s}} / p\right)^{R / C_{p}}$ is potential temperature, $T$ is temperature, $p$ is pressure, $\rho$ is density, $p_{\mathrm{s}}$ is standard pressure, $R / C_{p}=(\gamma-1) / \gamma, g$ is gravitational constant, and overbars and primes denote mean and perturbation quantities, respectively. The body forcing and heating terms on the right-hand sides of Eqs. (1)-(4) have spatial distributions given by $F_{x}(\mathbf{x}), F_{y}(\mathbf{x}), F_{z}(\mathbf{x})$, and $J(\mathbf{x})$ and can be any continuous functions. The temporal distribution of the source terms is assumed to have the form

$$
\mathscr{F}(t)=\frac{2}{\sigma_{t}} \begin{cases}\frac{1}{2}(1-\cos \hat{a} t) & \text { for } 0 \leqslant t \leqslant \sigma_{t}, \\ 0 & \text { for } t \geqslant \sigma_{t}\end{cases}
$$

and has a total duration $\sigma_{t}$. The frequency of the source terms is $\hat{a}=2 \pi n / \sigma_{t}$, where $n$ is the number of forcing cycles. Our application of this methodology to gravity wave generation by MCCs assumes $n=1$. We also assume $N=0.02 \mathrm{rad} \mathrm{s}^{-1}$ for this solution as well as for the ray tracing.

The solutions to Eqs. (1)-(5) are Eqs. (3.8)-(3.17) in VF and are obtained in terms of the Fourier transforms of the variables, e.g.,

$$
\begin{aligned}
& u(x, y, z, t) \\
& \quad=\frac{1}{(2 \pi)^{3}} \iiint \mathrm{e}^{-\mathrm{i} k x-\mathrm{i} l y-\mathrm{i} m z} \tilde{u}(k, l, m, t) \mathrm{d} k \mathrm{~d} l \mathrm{~d} m,
\end{aligned}
$$

where the tilde denotes a Fourier transform. For horizontal body forces, the solution after the forcing is finished is composed of a mean response and a gravity wave response, with the mean response independent of the temporal variability (i.e., $\left.\sigma_{t}\right)$ of the forcing. However, the gravity wave response depends sensitively on the temporal variability of the forcing. In particular, if the forcing is applied very slowly in time (e.g., $\sigma_{t} \rightarrow \infty$ ), there is no gravity wave response. The exact gravity wave solutions after the forcing is completed (i.e., $t \geqslant \sigma_{t}$ ) are

$$
\begin{aligned}
\tilde{u}_{\mathrm{GW}} & =-\frac{D m \hat{a}^{2}}{k_{H}^{2}} \\
& \times\left\{\left(k \omega A_{\mathrm{F}}-\frac{l f B_{\mathrm{F}}}{\omega}\right) \mathscr{S}-\left(l f A_{\mathrm{F}}+k B_{\mathrm{F}}\right) \mathscr{C}\right\}, \\
\tilde{v}_{\mathrm{GW}} & =-\frac{D m \hat{a}^{2}}{k_{H}^{2}} \\
& \times\left\{\left(k f A_{\mathrm{F}}-l B_{\mathrm{F}}\right) \mathscr{C}+\left(\frac{k f B_{\mathrm{F}}}{\omega}+l \omega A_{\mathrm{F}}\right) \mathscr{S}\right\},
\end{aligned}
$$

$\tilde{w}_{\mathrm{GW}}=D \hat{a}^{2}\left(A_{\mathrm{F}} \omega \mathscr{S}-B_{\mathrm{F}} \mathscr{C}\right)$,

$\tilde{\Theta}_{\mathrm{GW}}=D N^{2} \hat{a}^{2}\left\{A_{\mathrm{F}} \mathscr{C}+\frac{B_{\mathrm{F}} \mathscr{S}}{\omega}\right\}$

with the usual dispersion relation

$\omega^{2}=\left(k_{H}^{2} N^{2}+m^{2} f^{2}\right) / \mathbf{k}^{2}$.

In the above, $\Theta \equiv g \theta^{\prime} / \bar{\theta}, \mathscr{S} \equiv \sin \omega t+\sin \omega\left(\sigma_{t}-t\right), \mathscr{C} \equiv$ $\cos \omega t-\cos \omega\left(\sigma_{t}-t\right), D=1 /\left(\sigma_{t} \omega^{2}\left(\hat{a}^{2}-\omega^{2}\right)\right), k_{H}^{2}=k^{2}+$ $l^{2}, \mathbf{k}^{2}=k_{H}^{2}+m^{2}, k, l$, and $m$ are the zonal, meridional, and vertical wavenumbers, respectively, the subscript "GW" refers to the gravity wave portion of the solution, and

$A_{\mathrm{F}}=\tilde{F}_{z}-\frac{m}{\mathbf{k}^{2}}\left(k \tilde{F}_{x}+l \tilde{F}_{y}+m \tilde{F}_{z}\right)$,

$B_{\mathrm{F}}=\frac{k_{H}^{2} \tilde{J}}{\mathbf{k}^{2}}+\frac{m f}{\mathbf{k}^{2}}\left(l \tilde{F}_{x}-k \tilde{F}_{y}\right)$.

\subsubsection{Gravity wave spectra and cospectra}

The most general solutions reveal a complex dependence of the gravity wave spectra and cospectra on the various possible source terms and the spatial and temporal character of the source(s) (see VF for details). We present here simplified solutions for horizontal and vertical body forces in order to compare the gravity wave response to MCCs with our previous applications.

The gravity wave spectra and cospectra resulting from a zonal body force with $A_{\mathrm{F}}=-m k \tilde{F}_{x} / \mathbf{k}^{2}, B_{\mathrm{F}}=0$, and $f=0$ (assuming tropical convection) are

$\overline{\left|\tilde{u}_{\mathrm{GW}}\right|^{2}}=\frac{\xi}{2}\left(\frac{k^{4}}{k_{H}^{4}}\right)\left(1-\frac{\omega^{2}}{N^{2}}\right)^{2}\left|\tilde{F}_{x}\right|^{2}$, 
$\overline{\left|\tilde{v}_{\mathrm{GW}}\right|^{2}}=\frac{\xi}{2}\left(\frac{k^{2} l^{2}}{k_{H}^{4}}\right)\left(1-\frac{\omega^{2}}{N^{2}}\right)^{2}\left|\tilde{F}_{x}\right|^{2}$,

$\overline{\left|\tilde{w}_{\mathrm{GW}}\right|^{2}}=\frac{\xi}{2}\left(\frac{k^{2}}{k_{H}^{2}}\right)\left(\frac{\omega^{2}}{N^{2}}\right)\left(1-\frac{\omega^{2}}{N^{2}}\right)\left|\tilde{F}_{x}\right|^{2}$,

$\overline{\left|\tilde{\Theta}_{\mathrm{GW}}\right|^{2}}=N^{2} \frac{\xi}{2}\left(\frac{k^{2}}{k_{H}^{2}}\right)\left(1-\frac{\omega^{2}}{N^{2}}\right)\left|\tilde{F}_{x}\right|^{2}$,

$\overline{\tilde{u}_{\mathrm{GW}} \tilde{w}_{\mathrm{GW}}^{*}}=-\frac{\xi}{2}\left(\frac{k^{3} m}{k_{H}^{4}}\right)\left(\frac{\omega^{2}}{N^{2}}\right)\left(1-\frac{\omega^{2}}{N^{2}}\right)\left|\tilde{F}_{x}\right|^{2}$,

$\overline{\tilde{v}_{\mathrm{GW}} \tilde{w}_{\mathrm{GW}}^{*}}=-\frac{\xi}{2}\left(\frac{k^{2} l m}{k_{H}^{4}}\right)\left(\frac{\omega^{2}}{N^{2}}\right)\left(1-\frac{\omega^{2}}{N^{2}}\right)\left|\tilde{F}_{x}\right|^{2}$,

where the overbar represents a temporal average, and

$\xi=\frac{4 \hat{a}^{4} \sin ^{2}\left(\omega \sigma_{t} / 2\right)}{\left(\hat{a}^{2}-\omega^{2}\right)^{2} \omega^{2} \sigma_{t}^{2}}$.

Eq. (21) yields $\xi \simeq 1$ for fast or impulsive body forces and $\xi \simeq 1 / 4$ for $\sigma_{t}=\tau_{\mathrm{c}}$ at $\omega=\omega_{\mathrm{c}}$, where $\tau_{\mathrm{c}}=2 \pi / \omega_{\mathrm{c}}$ and we define a characteristic frequency $\omega_{\mathrm{c}}$ as the frequency obtained from the dispersion relation, Eq. (12), for the characteristic scales of the source (see VF).

The corresponding spectral energy density (see VF) is

$\overline{\mathscr{E}}=\frac{\xi \bar{\rho}}{2} \frac{k^{2}}{k_{H}^{2}}\left(1-\frac{\omega^{2}}{N^{2}}\right)\left|\tilde{F}_{x}\right|^{2} \rightarrow \frac{\xi \bar{\rho}}{4}\left(1-\frac{\omega^{2}}{N^{2}}\right)\left|\tilde{F}_{x}\right|^{2}$,

where the arrow indicates the solution when $l=k$. This is a realistic approximation when the spatial scales of the forcing have similar lengths in both the zonal and meridional directions, as expected for MCC gravity wave sources.

We note that the spectral energy density for a zonal body force peaks at approximately the characteristic spatial scales of the body force provided (1) that $\omega_{\mathrm{c}}$ is somewhat less than $N$ and (2) that the duration of the forcing is sufficiently short to prevent the prefactor $\xi$ from cutting off the spectrum for frequencies higher than $\hat{a}$. Provided that $\hat{a} \gg \omega_{\mathrm{c}}$, the resulting gravity wave energy spectrum does not depend on the duration of the forcing, $\sigma_{t}$. This is also true for the component horizontal velocity and potential temperature spectra. The vertical velocity spectrum, however, differs in that gravity waves with frequencies higher than the characteristic frequency are enhanced relative to gravity waves having the characteristic frequency. The ratio of vertical to horizontal velocity spectra when $l=k$ is

$\frac{\overline{\left|\tilde{w}_{\mathrm{GW}}\right|^{2}}}{\overline{\left|\tilde{u}_{\mathrm{GW}}\right|^{2}}}=\frac{2 \omega^{2} / N^{2}}{\left(1-\omega^{2} / N^{2}\right)}$,

and readily demonstrates the preferential occurrence of significant vertical motions at the highest frequencies for any source function.
Thus, a fast horizontal body force excites gravity waves that have spatial scales and frequencies associated with the spatial scales of the body force, but constrained by the forcing duration, with vertical velocities biased towards somewhat higher frequencies. Shown for reference as solid (dashed) lines in the upper panels of Fig. 1 are frequency and horizontal and vertical wavenumber spectra of horizontal (vertical) velocities arising from a zonal body force having a spatial distribution given by a Gaussian of the form

$$
F_{x}(\mathbf{x})=u_{0} \exp \left(-\left[\frac{\left(x-x_{0}\right)^{2}}{2 \sigma_{x}^{2}}+\frac{\left(y-y_{0}\right)^{2}}{2 \sigma_{y}^{2}}+\frac{\left(z-z_{0}\right)^{2}}{2 \sigma_{z}^{2}}\right]\right),
$$

with full width and depth of $\mathscr{D}_{x} \equiv 4.5 \sigma_{x}=10 \mathrm{~km}, \mathscr{D}_{y} \equiv$ $4.5 \sigma_{y}=10 \mathrm{~km}, \mathscr{D}_{z} \equiv 4.5 \sigma_{z}=5 \mathrm{~km}, u_{0}=100 \mathrm{~m} \mathrm{~s}^{-1}$, and a temporal distribution given by Eq. (6) with $\sigma_{t}=2 \tau_{\mathrm{c}} \simeq 1100 \mathrm{~s}$. Note that the dominant horizontal and vertical wavelengths are $\sim 40$ and $6.3 \mathrm{~km}$, respectively, for the horizontal velocity spectrum and only slightly closer in scale for the vertical velocity spectrum. The dominant wave periods for the horizontal and vertical velocity spectra are $\sim 26$ and $19 \mathrm{~min}$, respectively. For slow body forces (i.e., $\sigma_{t} \gg \tau_{\mathrm{c}}$ ), the horizontal wavelengths increase and the vertical wavelengths and wave frequencies decrease with increasing forcing duration.

The gravity wave spectra and cospectra resulting from a vertical body force with $A_{\mathrm{F}}=k_{H}^{2} \tilde{F}_{z} / \mathbf{k}^{2}, B_{\mathrm{F}}=0$, and $f=0$ are

$$
\begin{aligned}
& \overline{\left|\tilde{u}_{\mathrm{GW}}\right|^{2}}=\frac{\xi}{2}\left(\frac{k^{2}}{k_{H}^{2}}\right)\left(\frac{\omega^{2}}{N^{2}}\right)\left(1-\frac{\omega^{2}}{N^{2}}\right)\left|\tilde{F}_{z}\right|^{2}, \\
& \overline{\left|\tilde{v}_{\mathrm{GW}}\right|^{2}}=\frac{\xi}{2}\left(\frac{l^{2}}{k_{H}^{2}}\right)\left(\frac{\omega^{2}}{N^{2}}\right)\left(1-\frac{\omega^{2}}{N^{2}}\right)\left|\tilde{F}_{z}\right|^{2}, \\
& \overline{\left|\tilde{w}_{\mathrm{GW}}\right|^{2}}=\frac{\xi}{2}\left(\frac{\omega^{4}}{N^{4}}\right)\left|\tilde{F}_{z}\right|^{2}, \\
& \overline{\left|\tilde{\Theta}_{\mathrm{GW}}\right|^{2}}=N^{2} \frac{\xi}{2}\left(\frac{\omega^{2}}{N^{2}}\right)\left|\tilde{F}_{z}\right|^{2}, \\
& \overline{\tilde{u}_{\mathrm{GW}} \tilde{w}_{\mathrm{GW}}^{*}}=-\frac{\xi}{2}\left(\frac{k m}{k_{H}^{2}}\right)\left(\frac{\omega^{4}}{N^{4}}\right)\left|\tilde{F}_{z}\right|^{2}, \\
& \overline{\tilde{v}_{\mathrm{GW}} \tilde{w}_{\mathrm{GW}}^{*}}=-\frac{\xi}{2}\left(\frac{l m}{k_{H}^{2}}\right)\left(\frac{\omega^{4}}{N^{4}}\right)\left|\tilde{F}_{z}\right|^{2} .
\end{aligned}
$$

The corresponding spectral energy density for a vertical body force is

$\overline{\mathscr{E}}=\frac{\xi \bar{\rho}}{2}\left(\frac{\omega^{2}}{N^{2}}\right)\left|\tilde{F}_{z}\right|^{2}$,

which implies that the peak spectral energy density (and of the component spectra and cospectra) occurs not at the characteristic frequency of the vertical body force but at a frequency larger than the characteristic frequency (provided 

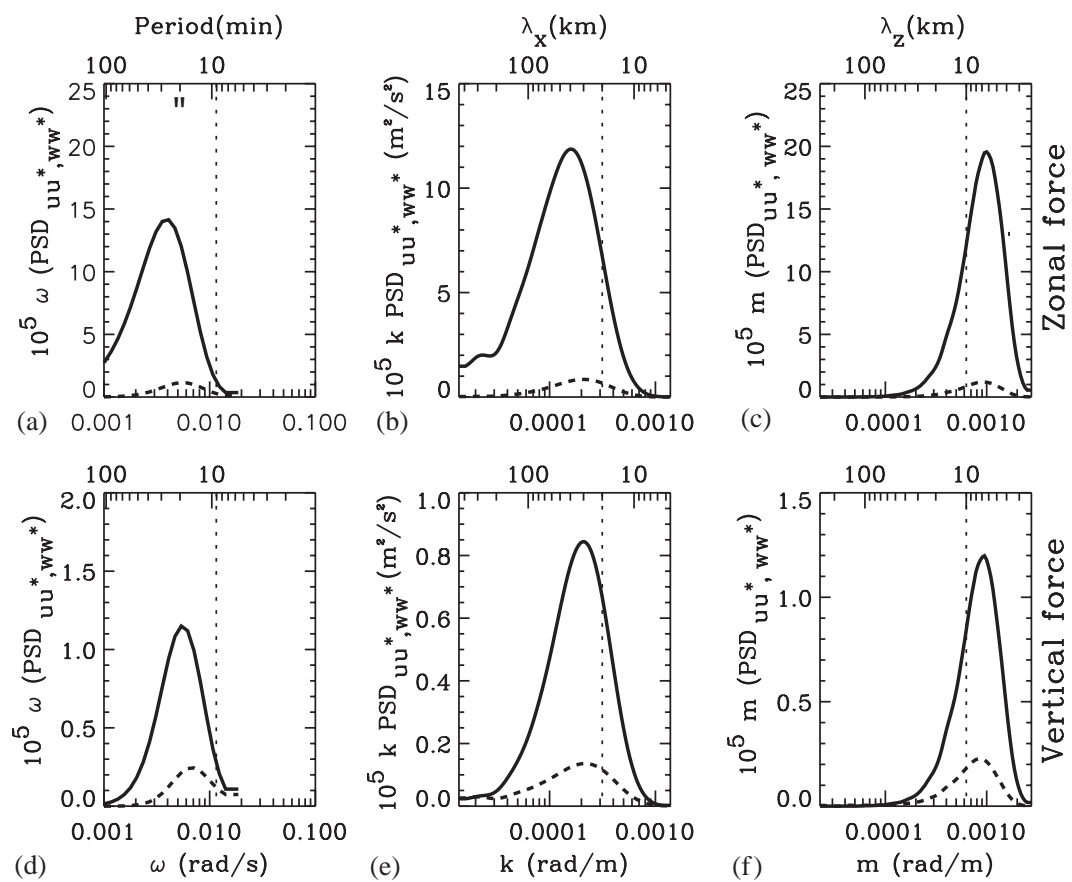

Fig. 1. Frequency (left) and horizontal (middle) and vertical (right) wavenumber spectra of horizontal (solid) and vertical (dash) winds for horizontal (top) and vertical (bottom) body forcing in variance content form following cessation of forcing. Other body force parameters were $u_{0}=100 \mathrm{~m} \mathrm{~s}^{-1}(\mathrm{a}-\mathrm{c}), w_{0}=100 \mathrm{~m} \mathrm{~s}^{-1}(\mathrm{~d}-\mathrm{f}), \mathscr{D}_{x}=\mathscr{D}_{y}=10 \mathrm{~km}, \mathscr{D}_{z}=5 \mathrm{~km}, f=0$, and $\sigma_{t}=2 \tau_{\mathrm{c}}$. The vertical dotted line in each panel indicates the characteristic frequency or scale (i.e., $\left.\omega_{c}, 2 \mathscr{D}_{x}, 2 \mathscr{D}_{z}\right)$ of the source.

the temporal duration of the forcing is sufficiently short) due to preferential excitation of the highest-frequency waves. Comparing these expressions with the gravity wave spectra and cospectra as well as the spectral energy density for a horizontal body force, Eqs. (15)-(20), (22), we observe that vertical body forces result in wave spectra that are fundamentally different from the wave spectra resulting from horizontal body forces. The ratio of vertical to horizontal velocity spectra obtained for a vertical body force is identical to that shown in Eq. (23) above. What is perhaps surprising is the ratios of vertical and horizontal velocity spectral amplitudes for horizontal and vertical body forces when the forcing geometries are the same, $\left|\tilde{F}_{x}\right|^{2}=\left|\tilde{F}_{z}\right|^{2}$. These are given by

$\frac{\overline{\left|\tilde{u}_{\mathrm{GW}}\right|_{\text {vert }}^{2}}}{\overline{\left|\tilde{u}_{\mathrm{GW}}\right|_{\text {horiz }}^{2}}}=\frac{\overline{\left|\tilde{w}_{\mathrm{GW}}\right|_{\text {vert }}^{2}}}{\overline{\left|\tilde{w}_{\mathrm{GW}}\right|_{\text {horiz }}^{2}}}=\frac{2 \omega^{2} / N^{2}}{\left(1-\omega^{2} / N^{2}\right)}$,

and are seen to have the same form as Eq. (23) in this special case. In general, however, gravity wave sources are not symmetric in the horizontal and vertical.

Shown in the lower panels of Fig. 1 are the horizontal and vertical velocity spectra arising from a vertical body force having the same spatial and temporal scales employed to compute the horizontal body force response spectra displayed in the upper panels, but with $w_{0}=100 \mathrm{~m} \mathrm{~s}^{-1}$ and

$$
F_{z}(\mathbf{x})=w_{0} \exp \left(-\left[\frac{\left(x-x_{0}\right)^{2}}{2 \sigma_{x}^{2}}+\frac{\left(y-y_{0}\right)^{2}}{2 \sigma_{y}^{2}}+\frac{\left(z-z_{0}\right)^{2}}{2 \sigma_{z}^{2}}\right]\right) .
$$

In this case the dominant horizontal and vertical wavelengths are $\sim 31$ and $7 \mathrm{~km}$, respectively, for the horizontal velocity spectrum and again slightly closer together for the vertical velocity spectrum. The associated dominant wave periods for the two spectra are $\sim 19$ and $15 \mathrm{~min}$, respectively, $\sim 30 \%$ shorter than found for the horizontal body force of the same geometry.

It is of interest to explore why horizontal and vertical body forces yield such different gravity wave spectra. Consider first a horizontal body force having a depth $\sigma_{z} \ll \sigma_{x}$ and $\sigma_{y}$. If such a body force is applied impulsively (Fritts and Luo, 1992; Luo and Fritts, 1993) or with $\sigma_{t} \lesssim \tau_{\mathrm{c}}$ in time (VF; Vadas and Fritts, 2002; Vadas et al., 2003), the gravity wave response is composed primarily of low-frequency waves having spatial scales dictated by the geometry of the body force. If this body force is instead applied slowly in time (i.e., $\sigma_{t} \gg \tau_{\mathrm{c}}$ ), the gravity wave response is even lower frequency (down to $f$ ), with much larger horizontal scales and somewhat smaller vertical scales than the source horizontal and vertical characteristic scales, respectively. The low-frequency motions and small vertical scales 
result because the source scales couple efficiently only to low-frequency motions; no large vertical motions are required to balance the slow and largely horizontal circulation induced by this body force geometry. When this same body force geometry is applied vertically and on a fast time scale, however, the result at first order is a body of fluid displaced largely vertically, induced to oscillate at frequencies much higher than the characteristic frequency (closer to the buoyancy frequency), and having a considerably greater depth than the depth of the body force because a largely horizontal balancing circulation, composed only of gravity waves, cannot occur on such rapid time scales. Thus the response in this case excites gravity waves not having the vertical source scales and characteristic frequencies, but having instead much deeper scales and much higher frequencies than implied by the source geometry alone.

The situation is very different for a deep body force having $\sigma_{z} \geqslant \sigma_{x}$ and $\sigma_{y}$. In this case, a horizontal body force acting slowly excites gravity waves having horizontal scales much larger than vertical scales (with vertical scales being somewhat smaller than source vertical scales, and horizontal scales being much larger than source horizontal scales), and having frequencies lower than the characteristic frequency when $\sigma_{z} \simeq \sigma_{x}$ and $\sigma_{y}$ and having frequencies of order the buoyancy frequency when $\sigma_{z} \gg \sigma_{x}$ and $\sigma_{y}$. These effects occur because, as above, largely horizontal motions are able to accommodate the required response. A fast forcing with this same body force geometry, in contrast, leads to gravity wave vertical scales comparable to vertical source scales because larger vertical motions (and higher wave frequencies) must play a role in the adjustment process. (In this case, the gravity wave horizontal scales are of order the source scales when $\sigma_{z} \simeq \sigma_{x}$ and $\sigma_{y}$, and are much greater than source scales when $\sigma_{z} \gg \sigma_{x}$ and $\sigma_{y}$.) Both lead to a mean response when the forcing is finished that has the same scales as the horizontal body force when $k=l$. On the other hand, a vertical body force of the same geometry leads only to gravity wave radiation, with the scales dictated by the forcing geometry for fast body forces. For slow body forces, the gravity wave vertical scales are somewhat smaller than vertical source scales, while the horizontal scales are much larger than horizontal source scales. The gravity wave frequency for slow body forces peaks at the buoyancy frequency for sources with $\sigma_{z} \gg \sigma_{x}$ and $\sigma_{y}$, and peaks at lower frequencies for sources with $\sigma_{z} \simeq \sigma_{x}$ and $\sigma_{y}$. We note that for all body forces, an important upper limit on the wave frequencies that can be efficiently excited is imposed by the source frequency $\hat{a}$, independent of the source geometry, because higher-frequency waves are not required to describe the induced circulation.

\subsection{Ray-tracing methodology}

Our ray-tracing model is built on the formalism developed by Lighthill (1978) and allows the wind, density, and other background parameters to change as functions of altitude and horizontal location. If a wave packet is propagating in a background wind, $\mathbf{V}(\mathbf{x})=\left(V_{1}, V_{2}, V_{3}\right)=(U, V, W)$, then its evolution in space and time is described by the following equations:

$\frac{\mathrm{d} x_{i}}{\mathrm{~d} t}=V_{i}+\frac{\partial \omega_{\mathrm{Ir}}}{\partial k_{i}}=V_{i}+c_{\mathrm{gi}}$

and

$\frac{\mathrm{d} k_{i}}{\mathrm{~d} t}=-k_{j} \frac{\partial V_{j}}{\partial x_{i}}-\frac{\partial \omega_{\mathrm{Ir}}}{\partial x_{i}}$

where the indices $i, j=1,2,3$ indicate the components of the vector quantities $\mathbf{x}, \mathbf{V}, \mathbf{k}$, and the group velocity $\mathbf{c}_{\mathrm{g}}$, repeated indices imply a summation, and $\omega_{\text {Ir }}$ is the real part of the intrinsic frequency of the gravity wave; $\omega_{\mathrm{Ir}}=\operatorname{real}(\omega)-k U-$ $l V-m W$, where real $(\omega)$ is the real part of the ground-based frequency.

For example, $\omega_{\text {Ir }}$ is given by

$\omega_{\mathrm{Ir}}^{2} \simeq \frac{k_{H}^{2} N^{2}}{m^{2}+k_{H}^{2}+1 / 4 H^{2}}$

in a fluid that has negligible viscosity and thermal conductivity, $f=0$, and for gravity waves that can be described by the anelastic approximation. Eq. (36) accurately represents the structure and behavior of the highest-frequency gravity waves in the mesosphere. As these waves propagate into the lower thermosphere, however, viscosity and thermal diffusivity become increasingly important with altitude due to the decreasing background density. Consequently, the dispersion relation must be modified to account for the role of dissipation in defining wave structure and propagation. Because the Prandtl number is $\mathrm{Pr} \approx 0.7$ throughout the mesosphere and lower thermosphere (Kundu, 1990), kinematic viscosity and thermal diffusivity will have comparable damping effects on a gravity wave at approximately the same altitude. To account for both of these effects, however, is beyond the scope of this paper (see Vadas and Fritts, 2004). Here, we consider only the role of molecular viscosity in damping the gravity waves.

The intrinsic frequency is defined as $\omega_{\mathrm{I}}=\omega-k U-l V$, where the horizontal wind is $(U, V, 0)$, where $\omega$ is the frequency with respect to the ground frame, and the perturbation solutions are proportional to $\exp (-\mathrm{i} \omega t)$ (e.g., the Fourier transforms of the zonal and vertical perturbation velocities of the gravity wave are $\tilde{u} \propto \exp (-\mathrm{i} \omega t)$ and $\tilde{w} \propto$ $\exp (-\mathrm{i} \omega t)$, respectively). The intrinsic frequency can be split into real and imaginary parts: $\omega_{\mathrm{I}}=\omega_{\mathrm{Ir}}+\mathrm{i} \omega_{\mathrm{II}}$. Here, $\omega_{\text {Ir }}$ is real and relates the intrinsic gravity wave frequency to the wave structure, buoyancy frequency, and damping due to kinematic viscosity, and $\omega_{\mathrm{Ii}}$ is real and expresses the inverse decay rate of the wave amplitude with time due to molecular viscosity. (The inverse rate of decay of the wave momentum flux is approximately twice $\omega_{\text {Ii }}$.) Using the Navier-Stokes momentum equations,

$\frac{\partial \mathbf{v}}{\partial t}+(\mathbf{v} \cdot \nabla) \mathbf{v}+\frac{1}{\rho} \nabla p-\mathbf{g}=v \nabla^{2} \mathbf{v}$ 
linearizing, making an anelastic approximation for the gravity waves, and assuming that $v$ is constant over a vertical wavelength at a given altitude $z$, the anelastic dispersion relation has the form (Vadas and Fritts, 2004)

$$
\begin{aligned}
\omega_{\mathrm{Ii}} & =-\frac{v}{2} \frac{\left(\mathbf{k}^{2}-1 / 4 H^{2}\right)}{(1+\delta / 2)} \\
\omega_{\mathrm{Ir}}^{2} & +\frac{v^{2}}{4}\left(\mathbf{k}^{2}-1 / 4 H^{2}\right)^{2} \frac{(1+\delta)}{(1+\delta / 2)^{2}}+\frac{v m \omega_{\mathrm{Ir}}}{H} \\
& =\frac{k_{H}^{2} N^{2}}{\mathbf{k}^{2}+1 / 4 H^{2}},
\end{aligned}
$$

where

$\delta=\frac{v m}{H \omega_{\text {Ir }}}$.

Note that $|\delta| \ll 1$ where the molecular viscosity is unimportant at lower altitudes. This dispersion relation is nonhydrostatic and compressible, but excludes acoustic waves, similar to Marks and Eckermann (1995), and yields the anelastic dispersion relation (Eq. (36)) when $v=0$ (and $\delta=0$ ). As a gravity wave propagates upward, wave damping increases, and the wave eventually reflects and dissipates strongly within a scale height where $\delta \simeq-1$. For waves with vertical wavelengths less than $4 \pi H, \omega_{\mathrm{Ii}}$ is always negative. To ensure that molecular viscosity causes dissipation even for waves with very large vertical wavelengths, we specify the inverse decay rate to be $-\left|\omega_{\mathrm{Ii}}\right|$. A gravity wave's momentum flux is then proportional to $\exp \left(-2\left|\omega_{\mathrm{Ii}}\right| t\right)$. In general applications, $N(z), H(z), \bar{\theta}(z)$, and $v(z)=\mu / \bar{\rho}(z)$ will all vary with altitude (and the presence of large-scale wave structures), placing additional restrictions on a formulation seeking more quantitative results. Here, we set $N=0.02 \mathrm{rad} \mathrm{s}^{-1}, H=7.0 \mathrm{~km}, \gamma=$ $1.4, \bar{T}=250 \mathrm{~K}, \mu=0.017 \mathrm{~g} \mathrm{~m}^{-1} \mathrm{~s}^{-1}, \bar{\rho}_{0}=10^{3} \mathrm{~g} \mathrm{~m}^{-3}$ and $\bar{\rho}=\bar{\rho}_{0} \exp (-z / H)$. This yields a kinematic viscosity at $90 \mathrm{~km}$ of $v=\mu / \bar{\rho}=6.5 \mathrm{~m}^{2} \mathrm{~s}^{-1}$, which is similar to the value used by Pitteway and Hines (1963) of $4 \mathrm{~m}^{2} \mathrm{~s}^{-1}$ at this altitude. We allow initially downward-propagating waves to reflect upwards at $z=0$. No other wave reflections at $z=0$ are allowed. In addition, we only allow waves with vertical wavelengths above the shear of $\lambda_{z} \leqslant 80 \mathrm{~km}$ to continue propagating upwards into the mesosphere/thermosphere in order to ensure that the anelastic approximation remains approximately valid. This follows the criteria that $|m|>1 / 2 H$, or $\lambda_{z}<4 \pi H=88 \mathrm{~km}$ for our assumed density scale height. In order to test the sensitivity of our results to this cutoff, we also performed the same calculations for the cutoff $\lambda_{z} \leqslant 100 \mathrm{~km}$ above the shear layer. We found that the results changed only slightly, implying not only that our results are robust, but also that it is the gravity waves with vertical wavelengths $<80 \mathrm{~km}$ above the shear that are most important to the thermospheric body forcing for our choices of shear and gravity wave MCC spectrum. To achieve accurate numerical solutions, a fourth-order Runge-Kutta routine (Press et al., 1992) is employed to advance the ray equations in time. Wave saturation effects are not included here, since the waves which dissipate at the highest altitudes have large enough phase speeds below the turbopause to likely avoid saturation (see Section 5.2). Results of this initial implementation of the viscous ray-tracing code are discussed in Section 5 below.

\section{Gravity waves arising due to MCCs}

We describe in this section the representation of MCC convection within our body force model and the characteristics of the initial wave field that arises from this source. We begin, however, by examining the impact on the resulting gravity wave spectra of variable spacings between multiple sources.

Fig. 2 shows frequency and horizontal wavenumber spectra of zonal wind for three identical vertical body forces that occur simultaneously and that have spatial and temporal scales of $\mathscr{D}_{x}=\mathscr{D}_{y}=10 \mathrm{~km}, \mathscr{D}_{z}=5 \mathrm{~km}, \sigma_{t}=1100 \mathrm{~s}$, and $w_{0}=100 \mathrm{~m} \mathrm{~s}^{-1}$ for horizontal forcing separations of $0,1,2$, and $3 \mathscr{D}_{x}$. The response for zero spacing is shown at $1 / 3$ amplitude because superposed forces lead to quadratically larger spectral amplitudes. The greatest departure of the spectra from multiple sources occurs for a source separation of only $1 \mathscr{D}_{x}$. In this case, such sources lead to dominant horizontal wavelengths at significantly larger scales than for a single source and a less obvious shift towards larger wave periods. For source separations of $2 \mathscr{D}_{x}$ or greater, however, horizontal wavelengths are peaked more strongly about that for a single source, with a reduction in the response at wavelengths of $\sim 50-100 \mathrm{~km}$ and an enhancement in the excitation of wavelengths larger than $\sim 200 \mathrm{~km}$. Despite these differences in the wavenumber spectra, frequency spectra for separations of $2 \mathscr{D}_{x}$ or larger are almost identical to that for a single source. Gravity wave spectra for larger source separations thus closely resemble the sum of spectra from individual sources, and we will utilize this fact in our description of the waves arising from MCC convection below.

To represent MCC convection, we generate 30 , spatially and temporally localized, Gaussian, vertical body forces (simulating convective plumes) that occur within an hour and over a small volume of the tropical upper troposphere. Because our model is linear, we cannot take into account wind shear effects on the generated gravity wave spectrum within the forcing area. The number of plumes is chosen to yield a mesoscale convective pattern similar to Lane et al. (2001), with individual plumes having differing diameters, depths, and strengths. Each plume has equal horizontal extents, $\sigma_{x}=\sigma_{y}$, with the full width and full depth of each plume $\mathscr{D}_{x}=4.5 \sigma_{x}$ and $\mathscr{D}_{z}=4.5 \sigma_{z}$, and a spatial distribution as defined by Eq. (33) above. The full width of each plume is chosen randomly between 10 and $20 \mathrm{~km}$ employing a boxcar probability distribution, $(10+10 \alpha) \mathrm{km}$, where $\alpha$ is a random number between 0 and 1 . The full depth, $\mathscr{D}_{z}$, is chosen to lie between 3 and $12 \mathrm{~km}$, but along a probability distribution that emphasizes $3 \mathrm{~km}$ over $12 \mathrm{~km}$ full depths, 

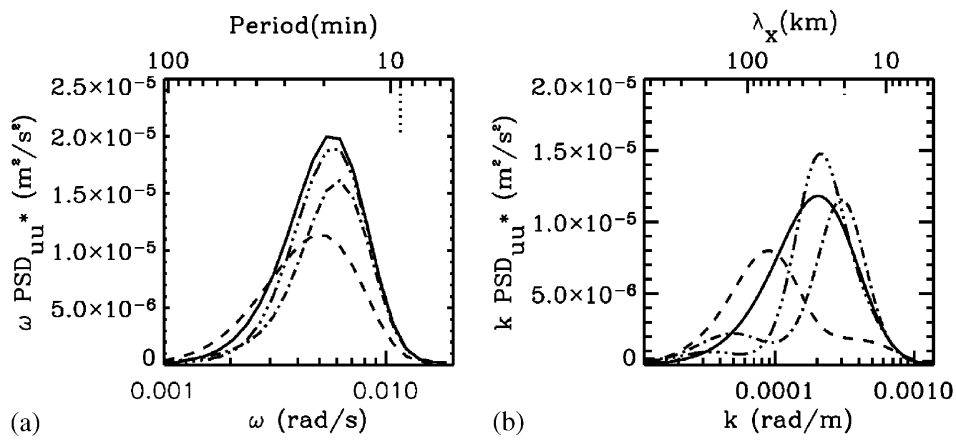

Fig. 2. Frequency (left) and zonal wavenumber (right) spectra of zonal wind in variance content form evaluated at the cessation of forcing for three vertical body forces spaced by 0 (solid), 1 (dash), 2 (dash-dot), and $3 \mathscr{D}_{x}$ (dash-dot-dot-dot lines) in the zonal direction. Each body force has $\mathscr{D}_{x}=\mathscr{D}_{y}=10 \mathrm{~km}, \mathscr{D}_{z}=5 \mathrm{~km}$, and $\sigma_{t}=2 \tau_{\mathrm{c}}$. The amplitude of the collocated forcing is reduced by 3 because the spectral response is quadratic in amplitude.
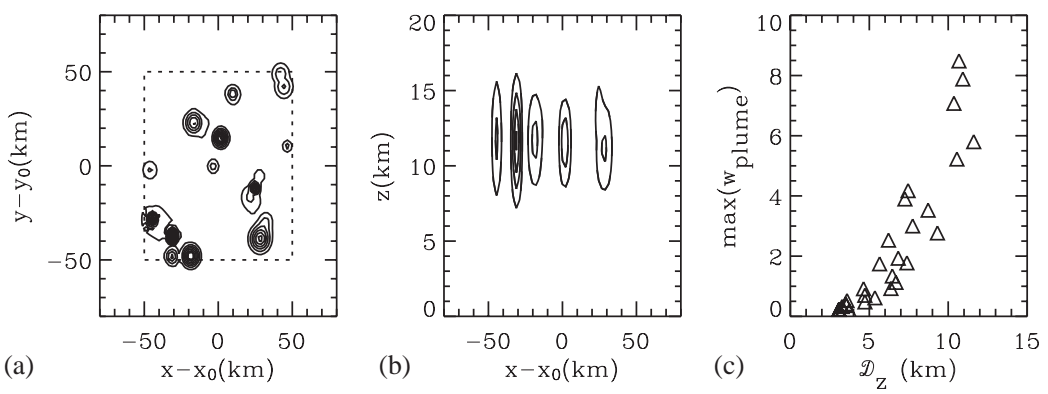

Fig. 3. Cross-sections of the maximum positive vertical velocities in a horizontal plane at $z=13 \mathrm{~km}$ (left) and in a vertical plane evaluated for all $y$ (middle). The contour lines show $0.5 \mathrm{~m} \mathrm{~s}^{-1}$ intervals. The right panel displays the maximum vertical velocities as a function of the body force depths. The dashed box in the left panel shows the region enclosing the plume centers.

$\mathscr{D}_{z}=\left(3+9 \alpha^{p}\right) \mathrm{km}$, where $p=2$. The strength of each plume is specified by

$w_{0}=w_{1}+\left(\sigma_{z}-\sigma_{z 1}\right) \frac{w_{2}-w_{1}}{\sigma_{z 2}-\sigma_{z 1}}$,

where $4.5 \sigma_{z 1}=3 \mathrm{~km}$ and $4.5 \sigma_{z 2}=12 \mathrm{~km}$. In addition, the vertical body force amplitude ranges from $w_{1}=50 \mathrm{~m} \mathrm{~s}^{-1}$ and $w_{2}=250 \mathrm{~m} \mathrm{~s}^{-1}$. It might be surprising that we are using such large force amplitudes. However, the 30 vertical body forces yield maximum vertical updraft velocities ranging from 0.1 to $8.5 \mathrm{~m} \mathrm{~s}^{-1}$. Thus, a vertical body force is largely balanced by potential temperature and pressure perturbations rather than by large vertical motions (see Eq. (3)).

Each plume center is chosen randomly to lie within $x_{0}=$ $[-50,50] \mathrm{km}$ and $y_{0}=[-50,50] \mathrm{km}$. Because gravity waves are generated most efficiently when convective plumes impact the stratosphere, we set the forcing altitude to be $z_{0}=z_{t r}-\mathscr{D}_{z} / 2$, where $z_{t r}=17 \mathrm{~km}$. The temporal behavior of each plume is given by Eq. (6) with a starting time chosen randomly within $t=[0,1] \mathrm{h}$. The duration of each plume, $\sigma_{t}$, is chosen to be

$\sigma_{t}=2 \tau_{\mathrm{c}}$, as in Figs. 1 and 2 above. The range of values of $\sigma_{t}$ for our choice of plume parameters is $13-46 \mathrm{~min}$.

Figs. 3a and show horizontal and vertical cross sections of the maximum positive plume velocities evaluated every $5 \min$ from $t=[0,1] \mathrm{h}$. Because of the probabilities assigned to strong and weak plumes, only a few plumes are deep and have large vertical motions. Because the smaller plumes are masked by the larger plumes in Fig. 3b, we also display the maximum updraft vertical velocities as a function of the body forcing depths for each body forcing in Fig. 3c. The largest body forcings have depths of $\sim 8-12 \mathrm{~km}$ and peak vertical velocities as large as $\sim 8.5 \mathrm{~m} \mathrm{~s}^{-1}$, and thus agree well with the convection simulations by Lane et al. (2001). Because gravity wave amplitudes are proportional to $w_{0}$ and frequencies are higher for deeper plumes, the few deepest plumes account for the large majority of MCC gravity wave variances and momentum fluxes. A sense of the temporal variability of the simulated plumes is provided in Fig 4, which displays horizontal cross sections of instantaneous vertical velocities at four times separated by $15 \mathrm{~min}$. These views illustrate highly transient behavior of the plumes. Each strong, deep updraft lasts for approximately 3-6 min, fol- 

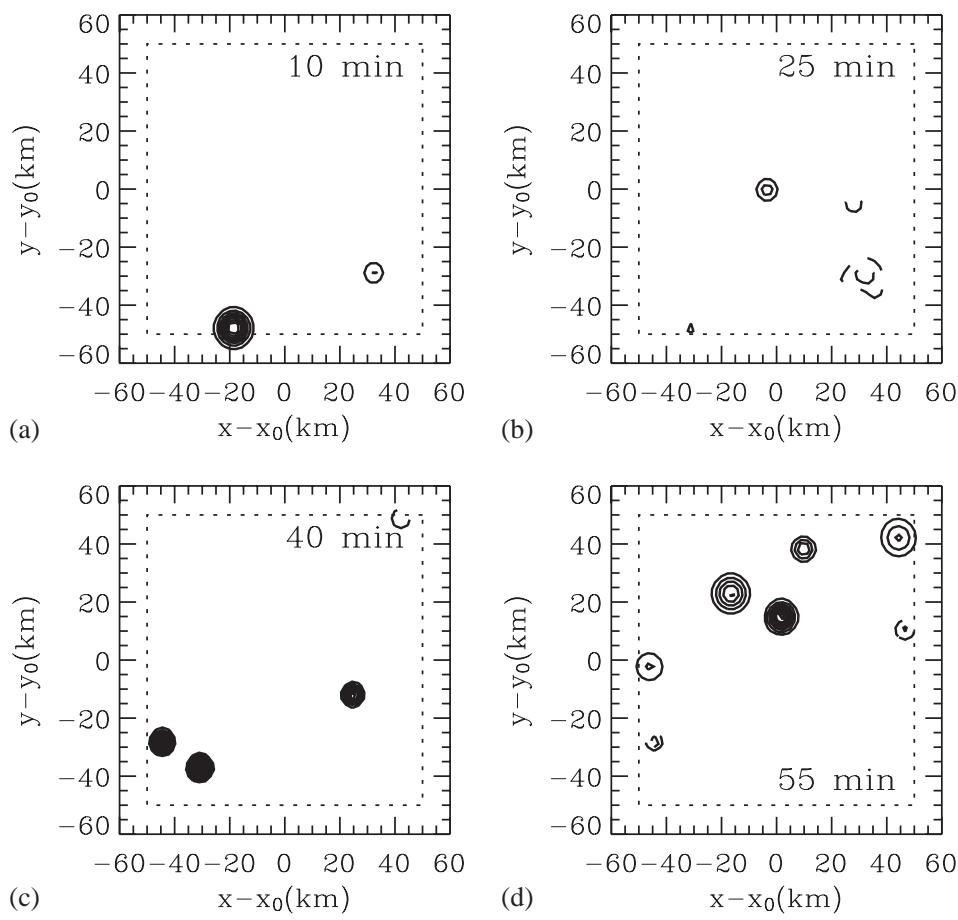

Fig. 4. Instantaneous vertical velocities in a horizontal plane at $13 \mathrm{~km}$ at 10 (a), 25 (b), 40 (c), and 55 (d) min during the period of convective excitation. The contour lines show $0.5 \mathrm{~m} \mathrm{~s}^{-1}$ intervals, with solid (dashed) lines denoting positive (negative) vertical velocities. As in Fig. 3, the dashed box shows the region enclosing the plume centers.

lowed a few minutes later by an equally strong downdraft (not shown). The plume with the strongest updraft is shown at nearly its peak amplitude in the lower left panel of Fig. 4 at $(x, y) \simeq(-31,-37) \mathrm{km}$. In addition, Fig. 4 shows that our assumption that the plumes are separated by at least their diameter is a good assumption in general. As above, this behavior was designed to replicate the temporal variability noted in $3 \mathrm{D}$ convective simulations and appears to be in reasonable agreement with those studies (Piani et al., 2000; Lane et al., 2001).

\section{Gravity wave propagation to higher altitudes}

We begin exploring gravity wave propagation above MCC convection by examining the wave field that arises from multiple plumes assuming the waves are not filtered or refracted by mean wind shears. This also provides a further comparison of our plume simulation with the results of 3D convective models in terms of wave amplitudes and scales.

Horizontal and vertical cross-sections of vertical velocity are shown in Fig. 5 at $t=30,60$, and 90 min following initiation of convective excitation. In order to include the compressible effect that the wave amplitudes increase with height from the density decreasing with height, we scale the wave amplitudes by $\sqrt{\bar{\rho}_{i} / \bar{\rho}}$, where $\bar{\rho}_{i}$ is the background density at the assumed initiation altitude of $z=14 \mathrm{~km}$. The horizontal cross-sections are at 25 and $50 \mathrm{~km}$ and the vertical cross-sections are at $y=0$. These reveal a wave field that is dominated by only a few of the deepest and strongest plumes. The major responses at $30 \mathrm{~min}$ have radii about their source plume of $\sim 30-50 \mathrm{~km}$ at $25 \mathrm{~km}$ and $\sim 50-100 \mathrm{~km}$ at $50 \mathrm{~km}$. Smaller radii correspond to the waves having the highest frequencies, smallest horizontal scales, and highest vertical group velocities, while larger radii at this time are responses to plumes that occurred somewhat earlier and were somewhat shallower, thus exciting lower-frequency waves.

The expanding wave fields observed at 60 and $90 \mathrm{~min}$ have two primary causes. The initial motion fields excited directly by convection at each altitude exhibit an evolution towards larger scales and larger radii, reflecting lower-frequency components of the wave spectrum having smaller vertical group velocities and requiring longer times to propagate to these altitudes. A second contributor to the later wave fields is that a portion of the initial response propagates downward and reflects from the Earth's surface, thus superposing higher- and lower- frequency wave motions that would otherwise occur in different regions at different times. At 90 min, we see an emergence, especially at $50 \mathrm{~km}$, of a more coherent and larger-scale response at the outer edges of the convective wave pattern that appears to be a merging of the faster responses to several of the stronger sources. 

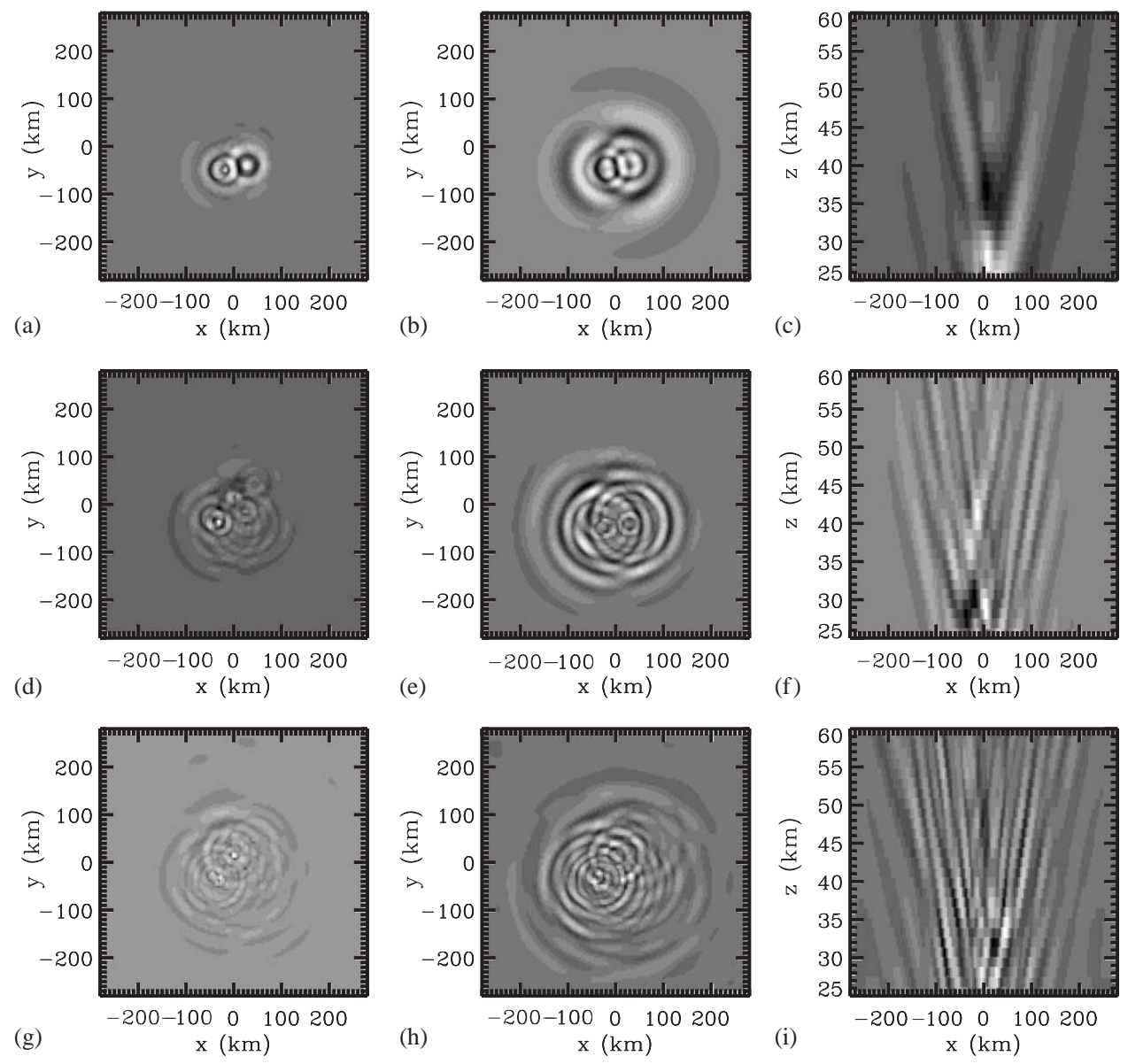

Fig. 5. Horizontal (left and center) and vertical (right) cross-sections of vertical velocity showing wave fields arising from simulated convective plumes at 30 (top), 60 (middle), and $90 \mathrm{~min}$ (bottom) after initiation of convective excitation. The left and center horizontal cross-sections are at altitudes of 25 and $50 \mathrm{~km}$, and the vertical $(x, z)$ cross-sections are at $y=0$. The broader wave fields seen at lower altitudes at later times are due both to the shallower propagation of lower-frequency waves having smaller vertical group velocities and to reflections from the Earth's surface of waves excited earlier and propagating downward initially. Maximum vertical velocities (scaled by $\sqrt{\bar{\rho}_{i} / \bar{\rho}}$ ) at 25 and $50 \mathrm{~km}$ are 0.86 and $1.2 \mathrm{~m} \mathrm{~s}^{-1}$ at $0.5 \mathrm{~h}, 1.5$ and $2.2 \mathrm{~m} \mathrm{~s}^{-1}$ at $1 \mathrm{~h}$, and 1.3 and $3.3 \mathrm{~m} \mathrm{~s}^{-1}$ at $1.5 \mathrm{~h}$. Here, $\bar{\rho}_{i}$ is the background density at the initiation altitude of $z=14 \mathrm{~km}$. The vertical cross-sections (right) show vertical velocities in the Boussinesq model. This can be thought of as the actual velocities weighted by $\sqrt{\bar{\rho} / \bar{\rho}_{i}}$ to offset the exponential increase in wave amplitudes arising from density decreases with altitude.

Similar responses, but at greater altitudes, are displayed in Fig. 6. Shown here are horizontal cross-sections of vertical velocity and perturbation temperature at $90 \mathrm{~km}$ (left and center panels, respectively) and vertical cross-sections of vertical velocity extending from 90 to $150 \mathrm{~km}$ at $y=0$ (right panels). The panels are at $t=30,60$, and $90 \mathrm{~min}$ from top to bottom. As in Fig. 5, we scale the wave amplitudes by $\sqrt{\bar{\rho}_{i} / \bar{\rho}}$. As at lower altitudes, the largest horizontal scales appear first because they have the largest associated vertical scales and vertical group velocities for the highest-frequency waves. At later times, smaller-scale motions appear at the same radii, implying common intrinsic frequencies but smaller vertical scales and vertical group velocities. Whereas multiple sources are evident in the vertical velocities at $25 \mathrm{~km}$ at 60 and $90 \mathrm{~min}$, the cross-sections at $90 \mathrm{~km}$ suggest only two dominant sources at early times, with other lesser sources (having smaller spatial scales, thus exciting waves with smaller scales and group velocities) having influences at later times. The vertical cross-sections reveal that the high-frequency, large-scale portion of the wave spectrum penetrates to very high altitudes very quickly.

To understand which components of these wave fields account for the largest forcing of the MLT, we display in Fig. 7 a 2D spectrum of the gravity wave momentum flux generated from our model of the MCC as a function of 

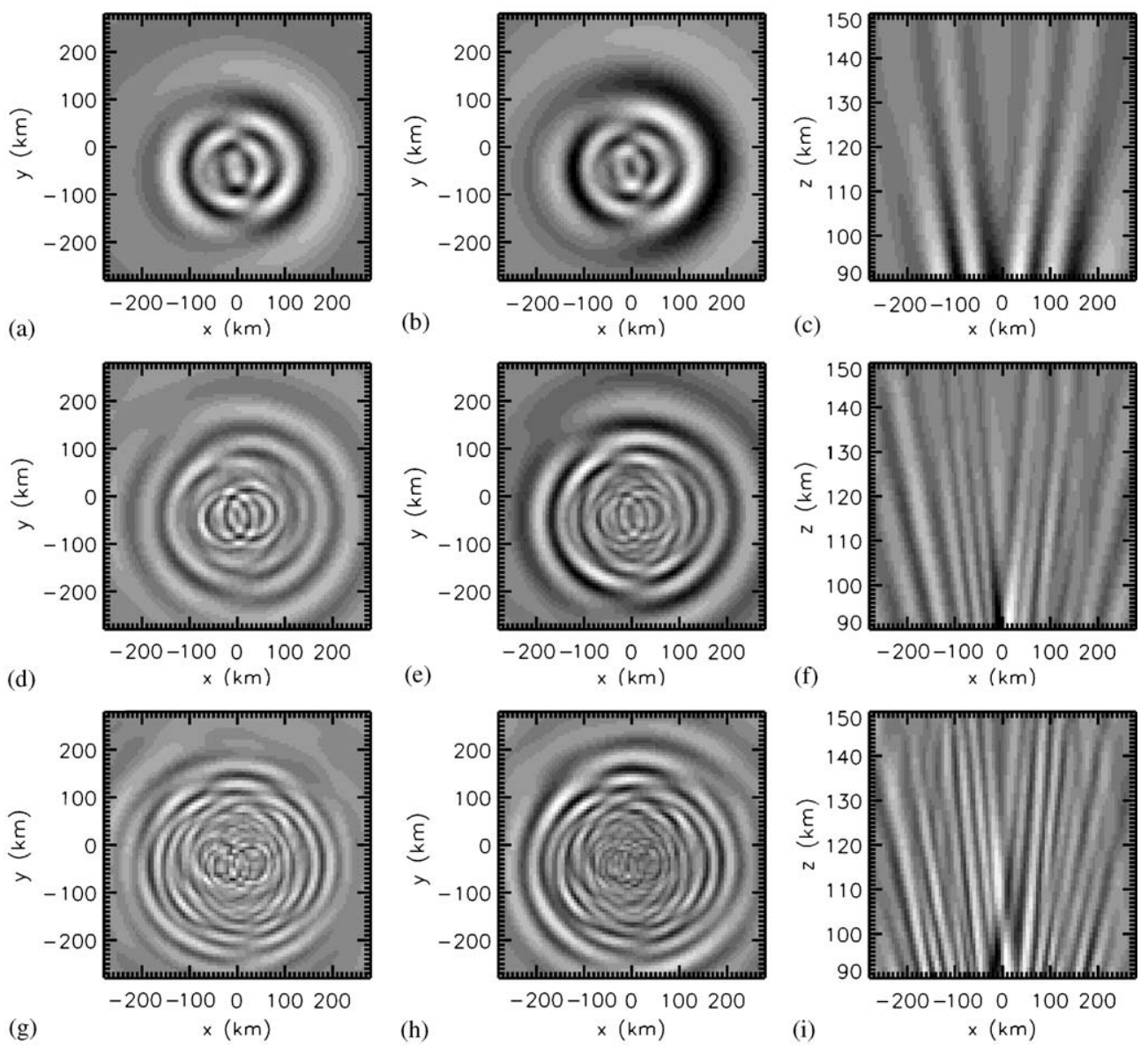

Fig. 6. As in Fig. 5, but with horizontal cross-sections of vertical velocity and perturbation temperature (left and center, respectively) at $90 \mathrm{~km}$ and vertical cross-sections of vertical velocity from 90 to $150 \mathrm{~km}$ at $y=0$ (right). Maximum vertical velocities $\left(\right.$ scaled by $\sqrt{\bar{\rho}_{i} / \bar{\rho}}$ ) are 3.5, 15, and $22 \mathrm{~m} \mathrm{~s}^{-1}$ and maximum temperature perturbations (scaled by $\sqrt{\bar{\rho}_{i} / \bar{\rho}}$ ) are 2.1, 8.6, and $16 \mathrm{~K}$, respectively. For reference, the maximum zonal velocities (scaled by $\sqrt{\bar{\rho}_{i} / \bar{\rho}}$ ) are $3.6,11$, and $21 \mathrm{~m} \mathrm{~s}^{-1}$ (not shown).

vertical wavelength and intrinsic frequency. This plot reveals a dominant contribution to the momentum flux at $\omega_{\text {Ir }} \sim 0.35 N, \lambda_{x} \sim 40 \mathrm{~km}$, and $\lambda_{z} \sim 15 \mathrm{~km}$, in line with expectations from our discussion above. Of these motions, those waves having the largest horizontal phase speeds and vertical group velocities above the stratospheric and mesospheric shears have the greatest potential for effects at the highest altitudes. Thus, the portion of the spectrum shown in Fig. 7 that contributes most at the highest altitudes depends sensitively on the shear at lower altitudes, as discussed in Section 5.

Overall, the wave fields discussed above resemble closely those modeled by Piani et al. (2000), Lane et al. (2001), and Horinouchi et al. (2002) and give us confidence that they are a sufficiently good approximation to gravity waves excited by MCC convection for our purposes in this paper. In particular, the scales and superposed patterns of the wave fields arising from multiple sources appear in close agree- ment. They do differ, however, in the degree of coherence of the wave fields at $90 \mathrm{~km}$ from the results of Horinouchi et al. (2002), suggesting either a less organized character of the convection in the direct numerical simulation results or the influences of mean shear on wave propagation and filtering that is not present in our results displayed in Figs. 5 and 6 . Our results also do not account for the effects of wave instability. However, peak vertical velocities exceeding $\sim 15 \mathrm{~m} \mathrm{~s}^{-1}$ and peak perturbation temperatures exceeding $\sim 9 \mathrm{~K}$ at $90 \mathrm{~km}$ at $60 \mathrm{~min}$ and beyond (in the absence of mean shear) imply wave amplitudes near the convective instability limit for the wave scales and intrinsic frequencies at which they occur. In reality, of course, gravity waves generated by convection will virtually always encounter wind shear throughout the MLT. Thus waves either having larger amplitudes or experiencing decreasing intrinsic phase speeds will likely become unstable in the MLT. But the larger amplitudes anticipated here to be important 


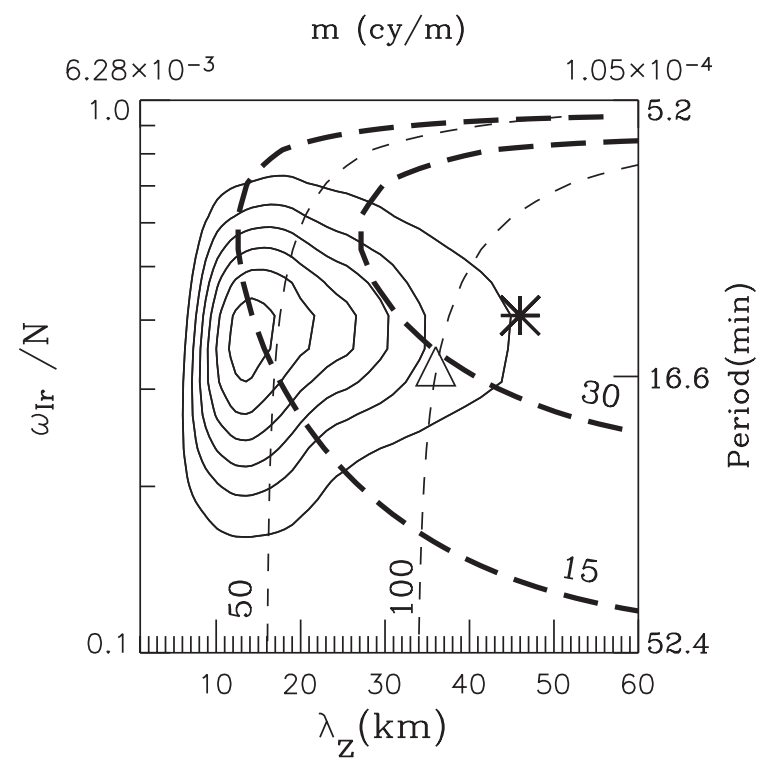

Fig. 7. Vertical flux of zonal momentum in flux content form as a function of vertical wavelength and $\omega_{\text {Ir }} / N$ for a single quadrant of wave propagation (i.e. upward and eastward, northward and southward) for all plume forcings (solid lines). Thick dashed lines indicate $c_{\mathrm{g} z}$ in $\mathrm{m} \mathrm{s}^{-1}$; light dash lines indicate $c_{x}$ when $l=0$ in $\mathrm{m} \mathrm{s}^{-1}$. The maximum response occurs at a frequency of $\omega_{\text {Ir }} \sim 0.35 N$ and a vertical wavelength of $\lambda_{z} \sim 15 \mathrm{~km}$. The asterisk shows those waves with $\lambda_{x}=103 \mathrm{~km}, l=0$, and $\lambda_{z}=46 \mathrm{~km}$. The triangle shows those waves with $\lambda_{x}=103 \mathrm{~km}, l=0$, and $\lambda_{z}=36 \mathrm{~km}$.

in the forcing of the thermosphere are likely not overturning below the turbopause because they are associated with larger vertical scales that have large phase speeds (as we will see in the following section). And those waves encountering critical levels are removed from the spectrum and play no role at higher altitudes. Due to the lack of evidence that turbulence plays a dissipative role above the turbopause (at $\sim 108 \mathrm{~km}$ altitude), saturation will probably be unimportant above the turbopause and will therefore likely not affect the amplitudes of the deepest waves with the highest phase speeds, as we argue in the following section. Because we do not include saturation in our ray-tracing model, those waves that would normally become unstable below the turbopause dissipate at altitudes below $150 \mathrm{~km}$. Because of the exponential growth of gravity wave momentum fluxes with altitude, this higher deposition of momentum flux will not affect our results for waves that dissipate at altitudes of 150-170 km (with our assumed thermospheric thermal structure), as we shall see in the next section. We explore filtering of this gravity wave spectrum by a mean wind shear below.

\section{Gravity wave filtering and thermospheric effects}

Gravity waves excited by MCC convection in the tropics encounter wind shears due to the quasi-biennial oscillation

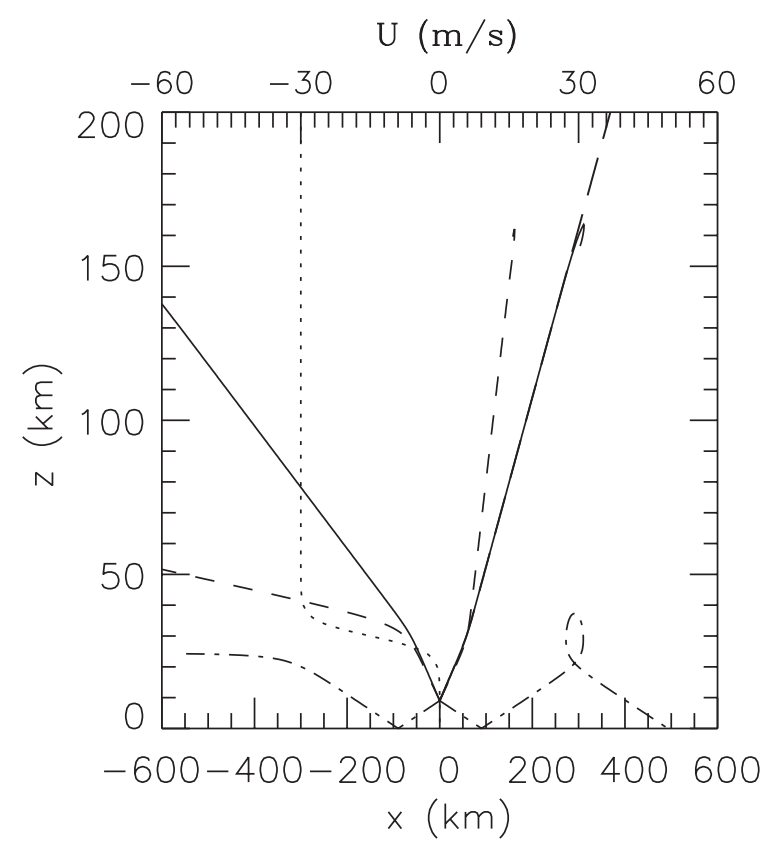

Fig. 8. Representative ray paths in the assumed mean zonal shear (dotted line) for two waves having $k= \pm 2 \pi / 103 \mathrm{~km}^{-1}, m=-2 \pi / 46 \mathrm{~km}^{-1}, c_{\mathrm{g} z}=37 \mathrm{~m} \mathrm{~s}^{-1}$, and $c_{x}= \pm 121 \mathrm{~m} \mathrm{~s}^{-1}$ (solid lines). Ray paths for two waves having $k= \pm 2 \pi / 45 \mathrm{~km}^{-1}, m=-2 \pi / 15 \mathrm{~km}^{-1}, c_{\mathrm{g} z}=13 \mathrm{~m} \mathrm{~s}^{-1}$, and $c_{x}= \pm 45 \mathrm{~m} \mathrm{~s}^{-1}$ (short dash lines). Ray paths for two waves having $k= \pm 2 \pi / 10 \mathrm{~km}^{-1}, m=2 \pi / 1 \mathrm{~km}^{-1}, c_{\mathrm{g} z}=-0.3 \mathrm{~m} \mathrm{~s}^{-1}$, and $c_{x}= \pm 3 \mathrm{~m} \mathrm{~s}^{-1}$ (dash-dot lines). Ray paths for two waves having $k= \pm 2 \pi / 20 \mathrm{~km}^{-1}, m=-2 \pi / 2 \mathrm{~km}^{-1}, c_{\mathrm{g} z}=0.6 \mathrm{~m} \mathrm{~s}^{-1}$, and $c_{x}= \pm 6 \mathrm{~m} \mathrm{~s}^{-1}$ (dash-dot-dot-dot lines). For reference, a ray path is shown for one wave having $k=2 \pi / 103 \mathrm{~km}^{-1}$ and $m=-2 \pi / 46 \mathrm{~km}^{-1}$ but with $v=0$ (light, long dash line). All waves originate at $z=9 \mathrm{~km}$ and $t=17 \mathrm{~min}$. Note the critical level response within the shear layer for the waves propagating westward with small phase speeds, and the refraction to higher and lower intrinsic frequencies of the waves transmitted through the shear layer and propagating to higher altitudes.

(QBO) in the lower stratosphere, the semiannual oscillation (SAO) near the stratopause and mesopause, various tidal and planetary wave structures increasing in amplitude with altitude, and other equatorial and gravity wave motions occurring on a wide range of scales. Thus, rather than specifying single or multiple wind fields that cannot span a reasonable set of representative cases, we consider the implications of a single shear layer of representative magnitudes. These are specified as a mean zonal wind of the form

$U=\frac{U_{0}}{2}\left(1+\tanh \left[\frac{z-z_{\mathrm{c}}}{z_{0}}\right]\right) \mathrm{m} \mathrm{s}^{-1}$,

with $U_{0}=-30 \mathrm{~m} \mathrm{~s}^{-1}$ (Figs. 8-15) or $U_{0}=-60 \mathrm{~m} \mathrm{~s}^{-1}$ (Figs. 16 and 17$), z_{\mathrm{c}}=30 \mathrm{~km}$, and $z_{0}=5 \mathrm{~km}$. Here, the mean wind decreases from zero to $-30 \mathrm{~m} \mathrm{~s}^{-1}$ for $U_{0}=-30 \mathrm{~m} \mathrm{~s}^{-1}$ (or zero to $-60 \mathrm{~m} \mathrm{~s}^{-1}$ for $U_{0}=-60 \mathrm{~m} \mathrm{~s}^{-1}$ ) between $\sim 24$ and 


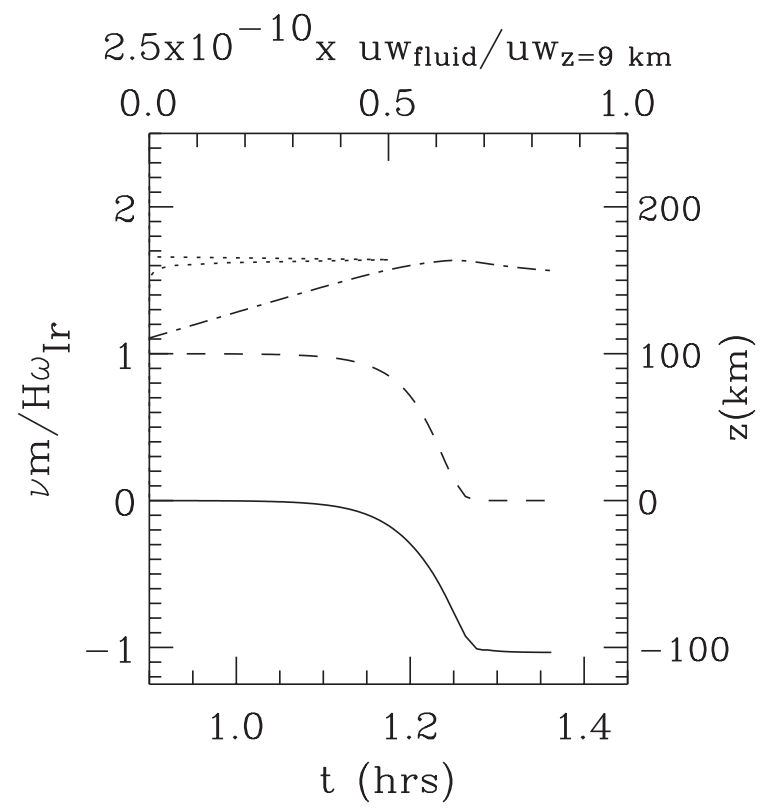

Fig. 9. For the eastward-propagating gravity wave represented by the solid line in Fig. 8, we show $\delta$ as a function of time (solid line, using the left axis scale), $\overline{u^{\prime} w^{\prime}} / \overline{u^{\prime} w^{\prime}}\left(z_{0}\right) \exp \left(-\left(z-z_{0}\right) / H\right)$ of the wave as a function of time (dash line, using the left axis scale), the altitude of the gravity wave as a function of time (dash-dot line, using the right axis scale), and the amount of momentum flux deposited into the background fluid divided by the gravity wave momentum flux at $z_{0}=9 \mathrm{~km}$ as a function of the altitude $z$ (dotted line, using the top and right axis scales).

$36 \mathrm{~km}$. The mean zonal wind for $U_{0}=-30 \mathrm{~m} \mathrm{~s}^{-1}$ is shown in Fig. 8 as a dotted line. The evolution of the MCC gravity wave spectrum in this environment is computed using our ray tracing methodology described above in Section 2.2.

\subsection{Ray paths in a mean shear}

Ray paths computed for several representative wave scales and frequencies are shown in Fig. 8 to illustrate the diversity of ray paths that arise in response to wind shear and increasing viscosity with altitude. Gravity waves propagating eastward acquire larger intrinsic phase speeds, $\omega_{\text {Ir }}=k\left(c_{x}-U\right)$, and are refracted to larger vertical wavelengths in passing through the zonal wind shear. For example, the eastward propagating gravity wave represented by a solid line initially has $\lambda_{x}=103 \mathrm{~km}, \lambda_{z}=46 \mathrm{~km}$, and $c_{\mathrm{g}, z}=37 \mathrm{~m} \mathrm{~s}^{-1}$. After passing through the shear, it has $\lambda_{x}=103 \mathrm{~km}, \lambda_{z}=67 \mathrm{~km}$, and $c_{\mathrm{g}, z}=49 \mathrm{~m} \mathrm{~s}^{-1}$. As long as the eastward propagating wave's frequency remains less than $N$, the wave emerges above the shear layer with a larger vertical wavelength, a steeper propagation angle, and a larger vertical group velocity (see the eastward propagating gravity waves shown by solid, short dash, and dash-dot-dot-dot lines). If, on the other hand, $\omega_{\text {Ir }}>N$ above the shear layer, the ray path becomes vertical at the altitude at which $\omega_{\text {Ir }}=N$ and the wave reflects from this "turning level" in a loop and is trapped at lower altitudes (see the eastward propagating wave shown by the dash-dot line).

Gravity waves propagating westward exhibit a decreasing intrinsic frequency upon encountering the mean wind shear (because $c_{x}$ and $U$ now have the same sign). If $c_{x}-U<0$ (or $\left|c_{x}\right|>|U|$ ), then the wave emerges above the shear layer with a reduced intrinsic frequency, a smaller vertical wavelength, a shallower ray path, and a smaller vertical group velocity (see the westward propagating gravity waves shown by solid and short dash lines). But if $\left|c_{x}\right|<|U|$, the wave encounters a critical level where $\omega_{\text {Ir }} \rightarrow 0$, and according to linear theory and numerical modeling results, it is dissipated and removed from the wave spectrum (see the westward propagating gravity waves shown by dash-dot and dashdot-dot-dot lines).

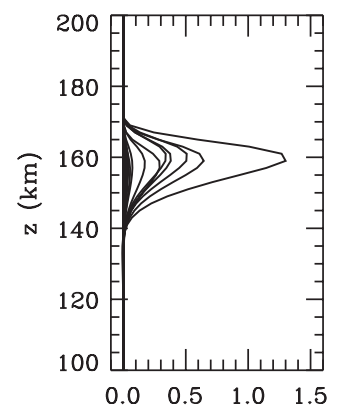

(a) $10^{-4} \quad \Sigma_{x, y, t}[\mathrm{uw}]\left(\mathrm{m}^{2} / \mathrm{s}^{2}\right)$

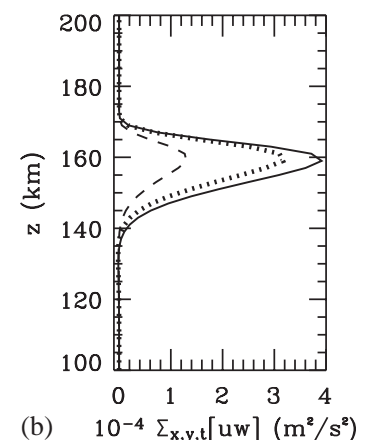

(b) $10^{-4} \Sigma_{x, y, t}[u w]\left(\mathrm{m}^{2} / \mathrm{s}^{2}\right)$

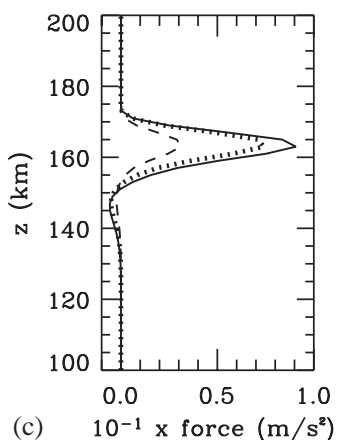

(c) $10^{-1} \mathrm{x}$ force $\left(\mathrm{m} / \mathrm{s}^{2}\right)$

Fig. 10. Vertical profiles of momentum flux for all individual plumes as solid lines (a), for the sum of all plumes as a solid line (b), and the body force arising from the dissipation of gravity waves from all plumes as a solid line (c). Also included in (b) and (c) are the distributions for the sum of the 5 deepest plumes only (dot lines), and the distributions for the deepest plume only (dash lines). All profiles are summed horizontally and temporally. 

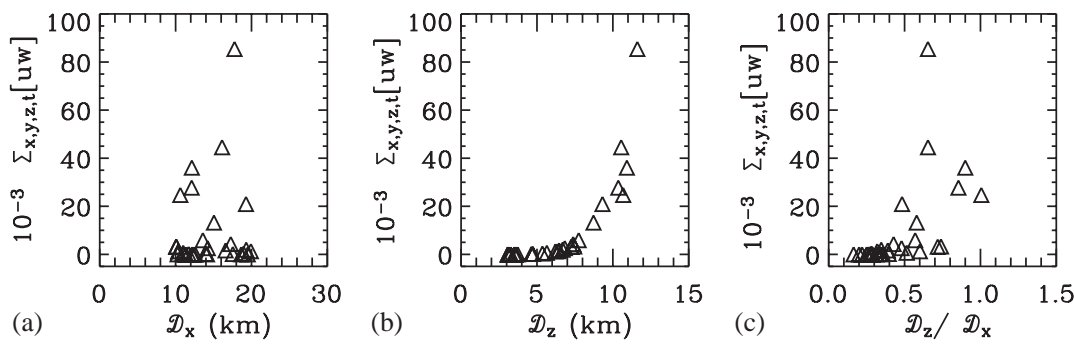

Fig. 11. Total deposited momentum flux for each plume as functions of plume full width (a), plume full depth (b), and ratio of plume depth to width (c). Note that the momentum flux correlates best with plume depth because the forcings with the greater depths are stronger and can excite deeper wavelength gravity waves.
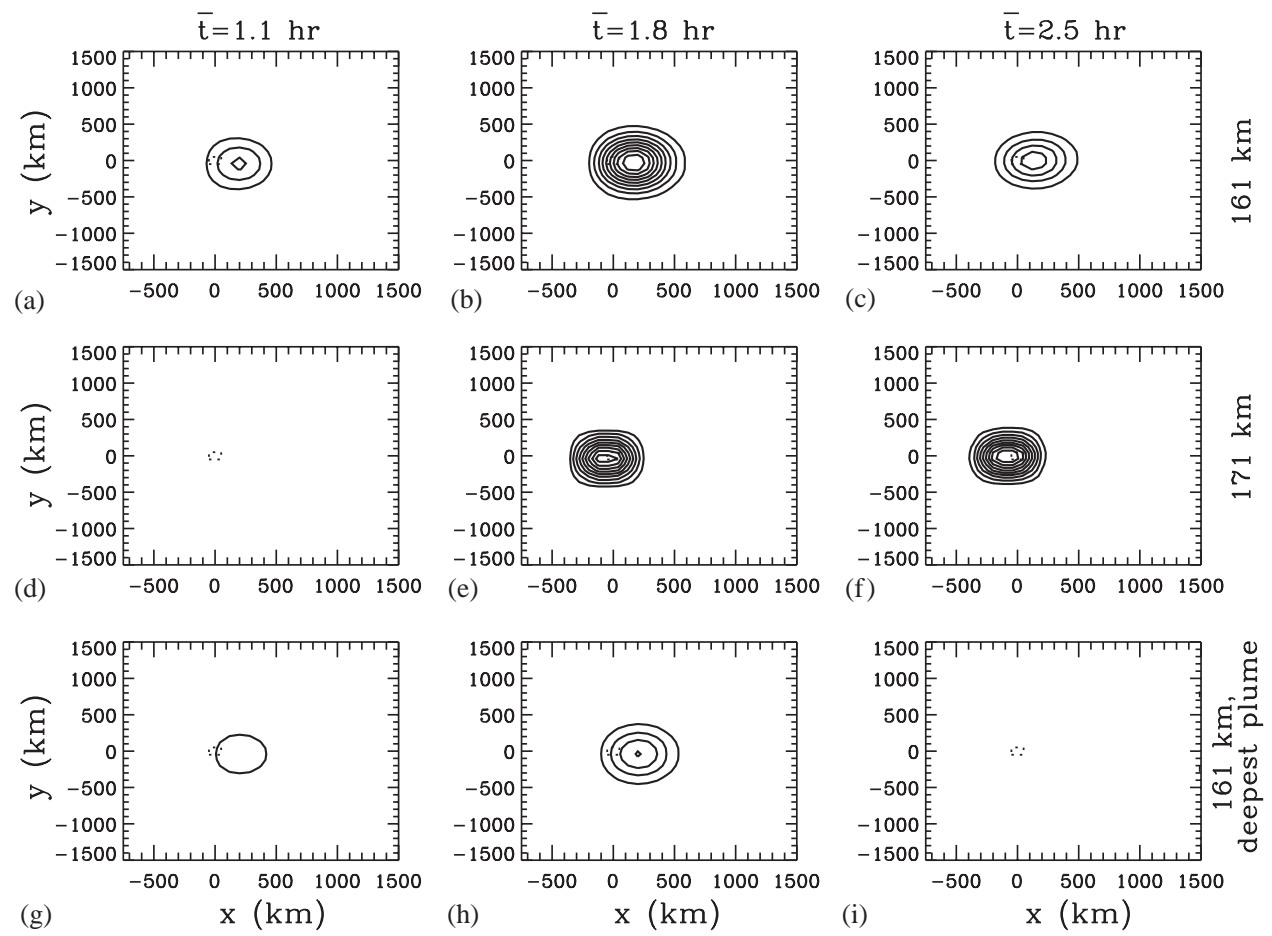

Fig. 12. Horizontal cross-sections of the total momentum flux in $2 \mathrm{~km}$ altitude bins deposited at $161 \mathrm{~km}(\mathrm{a}-\mathrm{c})$ and at $171 \mathrm{~km}(\mathrm{~d}-\mathrm{f})$ averaged over $\sim 30$-min bins centered at $1.1 \mathrm{~h}(\mathrm{a}, \mathrm{d}, \mathrm{g}), 1.8 \mathrm{~h}(\mathrm{~b}, \mathrm{e}, \mathrm{h})$, and $2.5 \mathrm{~h}(\mathrm{c}, \mathrm{f}, \mathrm{i})$. The momentum flux deposited by only the deepest plume is shown at these averaged times in $(\mathrm{g}-\mathrm{i})$ at $161 \mathrm{~km}$. The maxima values in rows 1,2 and 3 are $\simeq 200 \mathrm{~m}^{2} \mathrm{~s}^{-2}, \simeq 8 \times 10^{-5} \mathrm{~m}^{2} \mathrm{~s}^{-2}$, and $\simeq 80 \mathrm{~m}^{2} \mathrm{~s}^{-2}$, respectively. The scaling values in rows 1 and 2 are the maxima values for each row. The scaling value in row 3 is $\simeq 200 \mathrm{~m}^{2} \mathrm{~s}^{-2}$. Contours are $10 \%$ of the scaling value. The dotted boxes show the size of the MCC region.

Those eastward propagating gravity waves in Fig. 8 that refract in the shear flow but continue to propagate vertically at greater altitudes undergo further refraction to somewhat smaller vertical wavelengths near their dissipation level, which occurs as a result of the exponential increase in kinematic viscosity with increasing altitude. Ultimately, dissipation causes each ray to reflect at this viscous "turning level". However, at this point, $\delta \simeq-1$ and dissipation due to molecular viscosity is so strong that the wave typically travels only a fraction of a scale height below this turning level before the gravity wave loses essentially all of its momentum flux to the background flow. For example, the eastward traveling gravity wave represented by the solid line in Fig. 8 turns at $z \simeq 163 \mathrm{~km}$, and dissipates completely by $z \simeq 156 \mathrm{~km}$. This turning level altitude is highly dependent on the wave scales. These features are shown in Fig. 8, along with the path a gravity wave would take if there were no kinematic viscosity (light, long dashed line). 

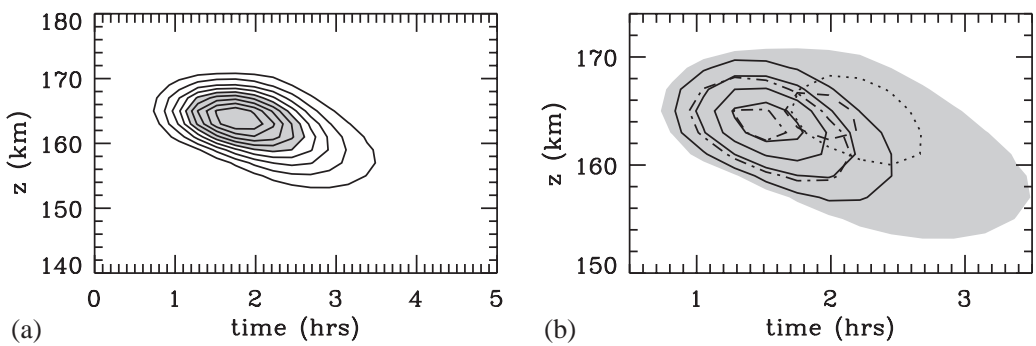

Fig. 13. (a) Vertical distribution of horizontally integrated induced thermospheric body forces at $+10 \%$ intervals of the maximum value of $1.5 \mathrm{~m} \mathrm{~s}^{-2}$ (solid lines). The shaded area depicts momentum fluxes greater than $50 \%$ of this maximum value. Negative values are less than $10 \%$ of this maximum value. The peak deposition occurs from $\sim 1$ to $3 \mathrm{~h}$. (b) A close-up image showing the composition of the contributions to (a). The shading indicates the total body force for positive values greater than $10 \%$ of the maximum value. The solid, dot, dash, dash-dot, and dash-dot-dot-dot lines indicate the positive portion of the induced body forces for the 5 deepest convective plumes, respectively, normalized by the same maximum.

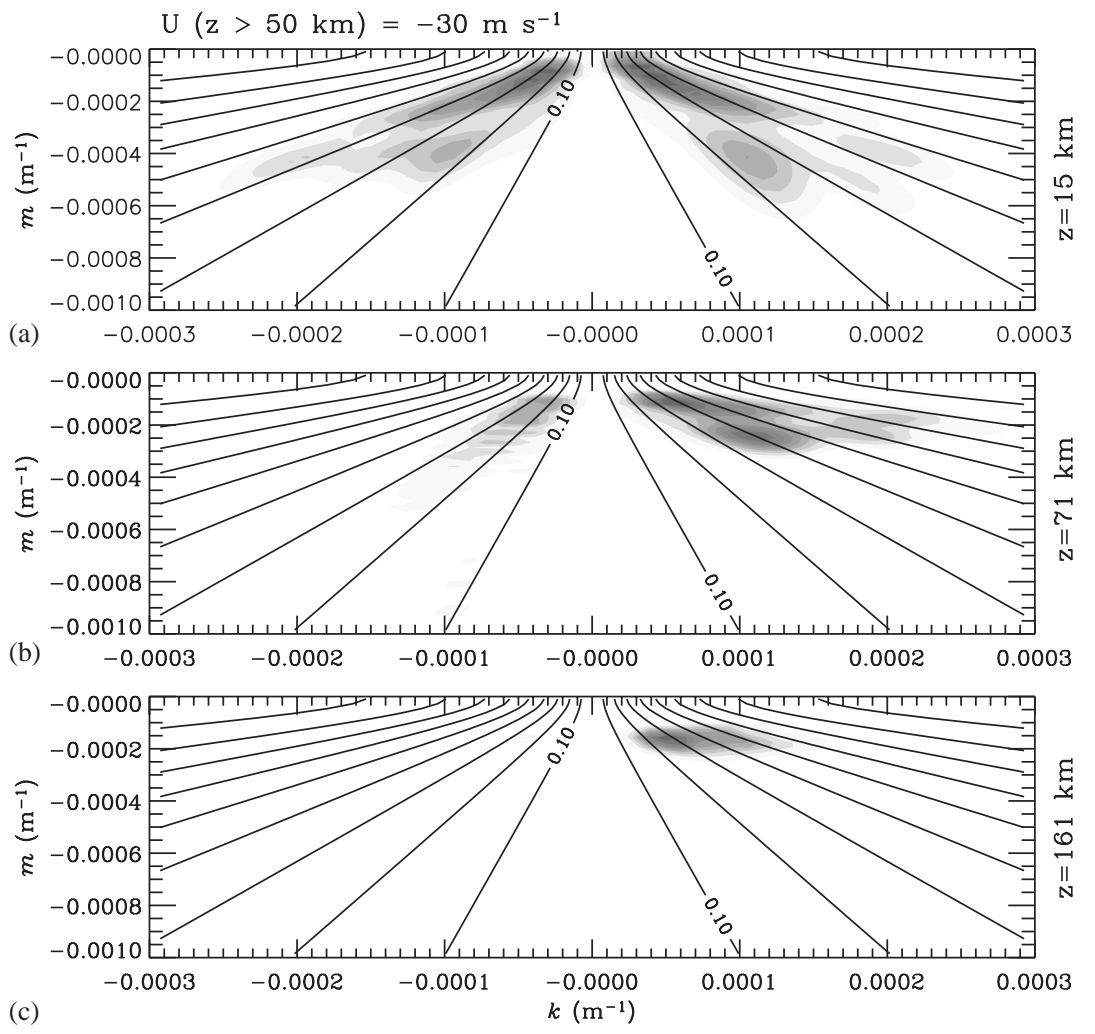

Fig. 14. Gravity wave momentum fluxes as a function of the horizontal and vertical wavenumbers at differing altitudes for $U_{0}=-30 \mathrm{~m} \mathrm{~s}$ (shading). The initial spectrum of gravity waves (shown in Fig. 7) is released at $x=0, y=0, z=13 \mathrm{~km}$, and $t=0.5 \mathrm{~h}$, and includes only the initially upward-propagating waves with $l=0$. Altitudes shown are (a) $z=15 \mathrm{~km}$, (b) $z=71 \mathrm{~km}$, and (c) $z=161 \mathrm{~km}$. The gravity wave spectrum at each altitude includes contributions from the gravity waves as they propagate upwards. Each wave is integrated out to $t=50 \mathrm{~h}$, unless it encounters a critical level, reflects at $z=0$, or dissipates. Solid lines of constant intrinsic frequency, $\omega_{\mathrm{Ir}}$, divided by the buoyancy frequency, $N$, are shown in intervals of 0.1 (solid lines).

In Fig. 9, we display the quantity $\delta$ for the eastwardpropagating gravity wave shown by the solid line in Fig. 8. This quantity is approximately zero except within a few scale heights below the turning level, where it decreases towards -1 . After reaching the turning level, $\delta \simeq-1$ and remains at this value as the wave reflects and dissipates rapidly 


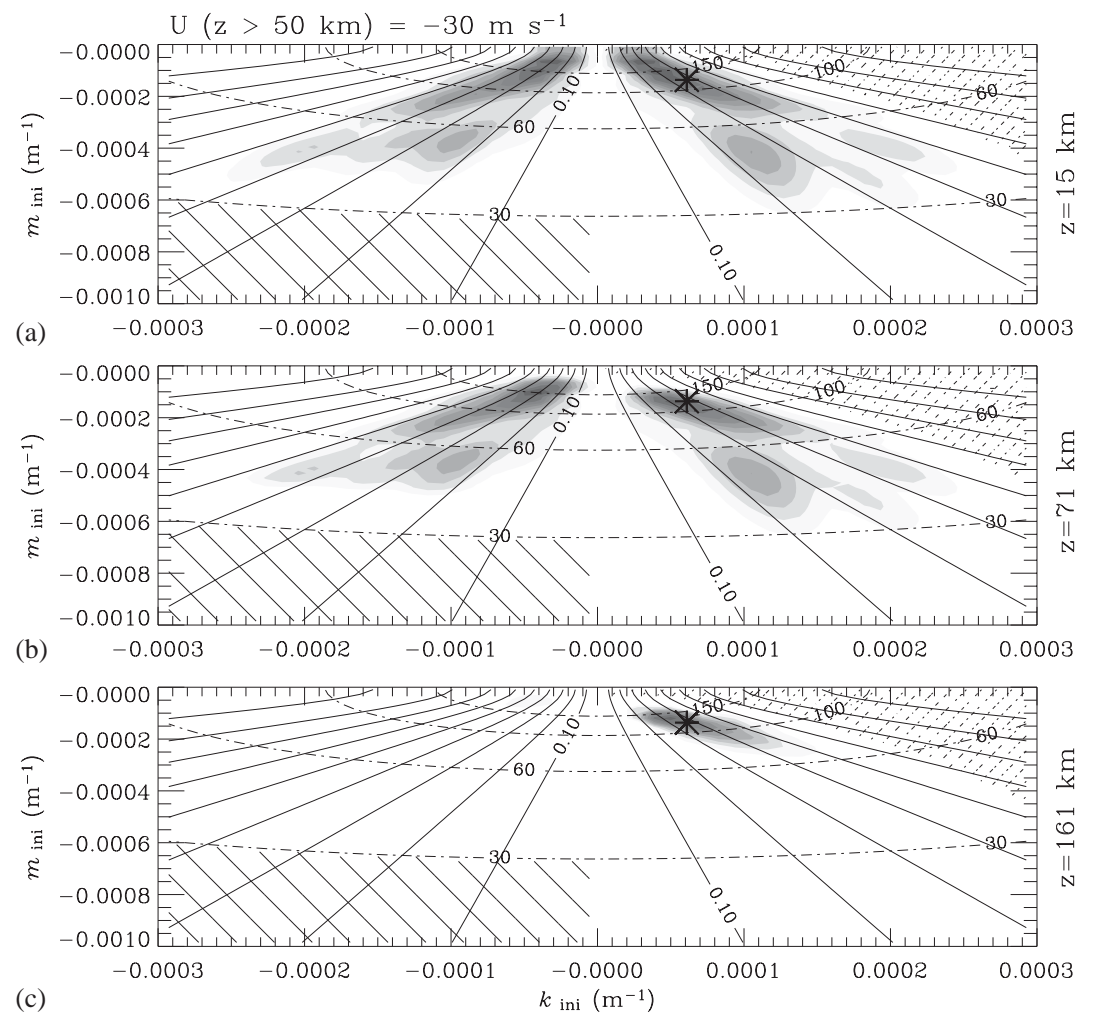

Fig. 15. Same as in Fig. 14, but as a function of the initial MCC horizontal and vertical wavenumbers. (The initial wavenumbers are those a gravity wave has when generated at $x=0, y=0, z=13 \mathrm{~km}$ and $t=0.5 \mathrm{~h}$.) The asterisk shows those waves with $\lambda_{x, \text { ini }}=103 \mathrm{~km}$ and $\lambda_{z, \text { ini }}=46 \mathrm{~km}$. Solid lines of frequency with respect to the ground frame of reference, $\omega_{r}$, divided by $N$ are shown in intervals of 0.1 . We also show the absolute value of the horizontal phase speed with respect to the ground frame of reference via dash-dot lines at $\left|c_{x}\right|=[30,60,100,150] \mathrm{m} \mathrm{s}^{-1}$. The waves eliminated by critical levels are shown as filled-in dash lines in the lower left corners of each panel (see Eq. (45)). The waves eliminated by evanescence in the shear layer are shown as filled-in dotted lines in the upper right corners of each panel (see Eq. (46)).

below the turning level. We also show $\overline{u^{\prime} w^{\prime}}(z) \exp ((z-$ $\left.\left.z_{0}\right) / H\right) \widehat{\overline{u^{\prime} w^{\prime}}}\left(z_{0}\right)$ as a function of time, the gravity wave altitude as a function of time, and the gravity wave momentum flux deposited into the fluid as a function of altitude. For this wave, by the time $|\delta|$ is large (i.e., $\delta \simeq-0.5$ ), the gravity wave has already lost $\sim 65 \%$ of its momentum flux as compared to a wave that encounters no kinematic viscosity. This loss is significant, and occurs by $z \sim 162 \mathrm{~km}$. The maximum momentum deposition occurs at the turning level $(z \sim 163 \mathrm{~km})$. The entire wave dissipation process takes approximately $15 \mathrm{~min}$. Note that the momentum fluxes (per unit mass) in the thermosphere are a few times $10^{9}$ times as large as the wave's momentum fluxes at $z_{0}=9 \mathrm{~km}$.

\subsection{Differential filtering and body forcing}

Gravity wave refraction in the zonal wind shear at lower altitudes removes waves from the spectrum penetrating to higher altitudes that encounter either turning levels or critical levels within the shear layer, as discussed above. The waves that emerge above the shear layer are refracted to higher or lower intrinsic frequencies, depending on whether they propagate eastward or westward (for our choice of mean zonal wind). This causes those waves propagating eastward to penetrate preferentially to higher altitudes before they are dissipated. The result is dissipation of westward-propagating waves (and small westward body forces, because of larger mean densities) at lower altitudes and later times, and dissipation of eastward-propagating waves (and large eastward body forces, because of smaller mean densities) at higher altitudes and earlier times. The altitude profiles of momentum flux for the wave spectrum arising from each convective plume, for the MCC as a whole, and the body forcing accompanying wave dissipation are displayed in Fig. 10. The body forcing shown in Fig. 10c is calculated via

$F_{\mathrm{b}}=-\frac{1}{\rho} \frac{\partial\left(\bar{\rho} \overline{u^{\prime} w^{\prime}}\right)}{\partial z}$.

In this case, horizontal and temporal averaging are performed on the momentum fluxes prior to calculating this 

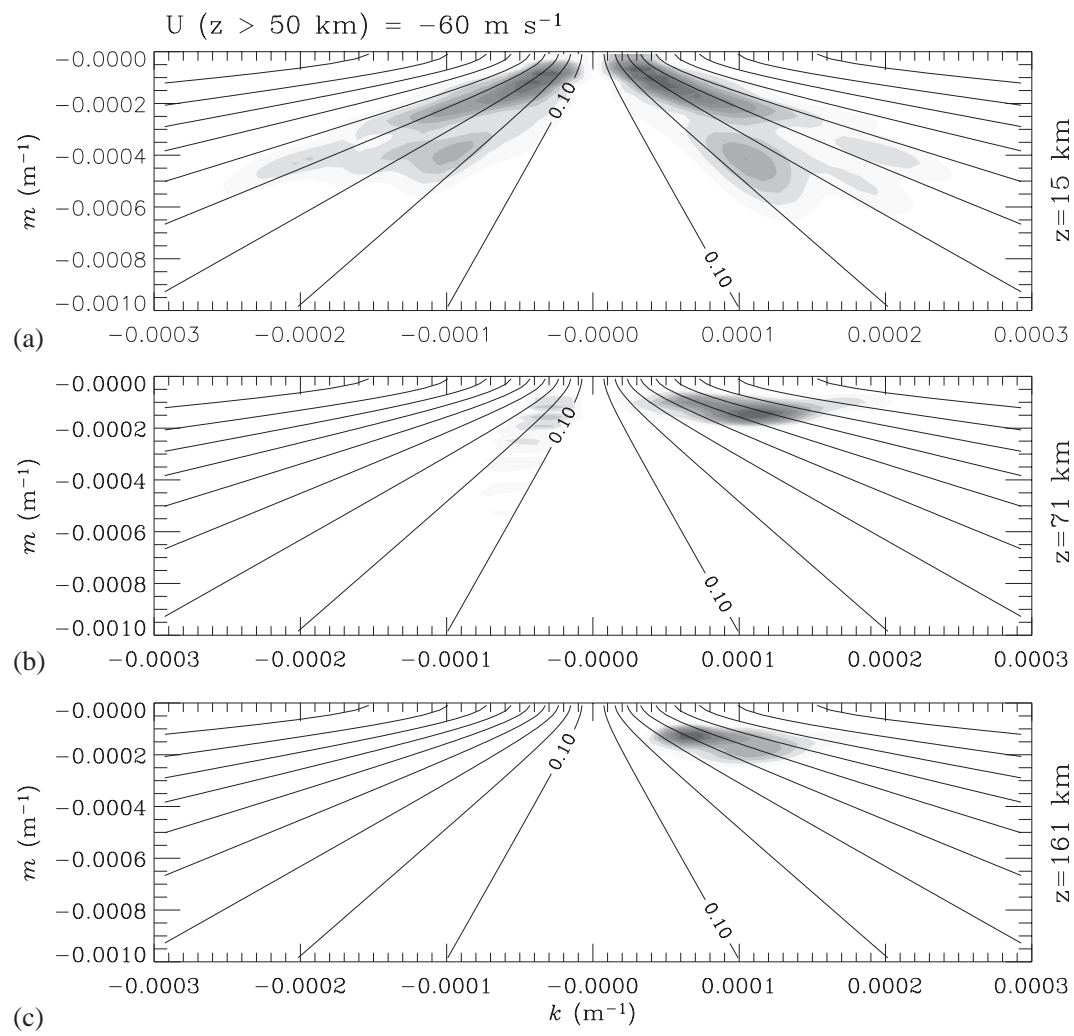

Fig. 16. Same as in Fig. 14, but for a background wind with $U_{0}=-60 \mathrm{~m} \mathrm{~s}^{-1}$. Here, the background zonal wind decreases from 0 to $-60 \mathrm{~m} \mathrm{~s}^{-1}$ between $\sim 24$ and $36 \mathrm{~km}$.

vertical derivative. In order to generate this figure (and Figs. 11-13), the gravity waves for each plume are assumed to be generated at the center of each body force at the time when that body force is a maximum. This assumption that a body force acts like a point source generator of gravity waves is motivated by Figs. 5 and 6. For Figs. 10-13, the normalizing volume $V$ we use to calculate the thermospheric momentum fluxes and forces is $V=1000 \mathrm{~km} \times 1000 \mathrm{~km} \times 25 \mathrm{~km}$ in the $x, y$, and $z$ directions, respectively. Here, the horizontal scales are chosen from Fig. 12 and the vertical scale is chosen from Fig. 10b. Each momentum flux profile is spread over a few scale heights, while the total momentum flux profile is spread over $\sim 3$ scale heights. Due to the point-particle assumption inherent when ray tracing, and to the deep vertical wavelengths of the waves which lead to these thermospheric forcings, we expect the thermospheric vertical profiles to be somewhat more spread in altitude than that depicted in Fig 10. Thus, the profiles in Fig. 10 are intended to represent the approximate center of the distribution. Due to the exponentially increasing viscosity with altitude, these waves would likely not be able to penetrate much further in altitude. And because even a scale height lower in altitude leads to a momentum flux amplitude reduction by $\mathrm{e}^{-1}$, we do not expect the momentum flux profile to extend very much lower in altitude than that shown in Fig. 10. For this shear profile, the momentum flux profile is maximum at altitudes of $z \sim 155-165 \mathrm{~km}$, while the body force profile is maximum at somewhat higher altitudes of $z \sim 155-170 \mathrm{~km}$. We also show in Fig. 10 the momentum fluxes and resultant body forcings that arise from the deepest as well as the 5 deepest convective plumes. It is observed that the 5 deepest plumes result in nearly all of the momentum flux and resultant thermospheric body forces, while the deepest convective plume contributes $\sim 33 \%$ to the total momentum flux and thermospheric body forcing.

We show in Fig. 11 the variations of the total deposited thermospheric momentum flux arising from each plume source versus the width of the plume, the depth of the plume, and the ratio of the depth to the width (a measure of the characteristic intrinsic frequency of the plume wave spectrum). These results reveal the dominant responses to be a consequence of deep, rather than steep, forcings, as these are the plumes having the largest forcing amplitudes and exciting gravity waves with the largest vertical wavelengths. Again, the plume with the largest thermospheric response is also the deepest plume, and the 5 deepest plumes create the 5 largest thermospheric responses.

The horizontal distribution of this deposited momentum flux is shown in Fig. 12 at $z=161 \mathrm{~km}(\mathrm{a}-\mathrm{c})$ and at $z=171 \mathrm{~km}$ (d-f) and at average times of $\bar{t}=1.1,1.8$, and $2.5 \mathrm{~h}$ after 

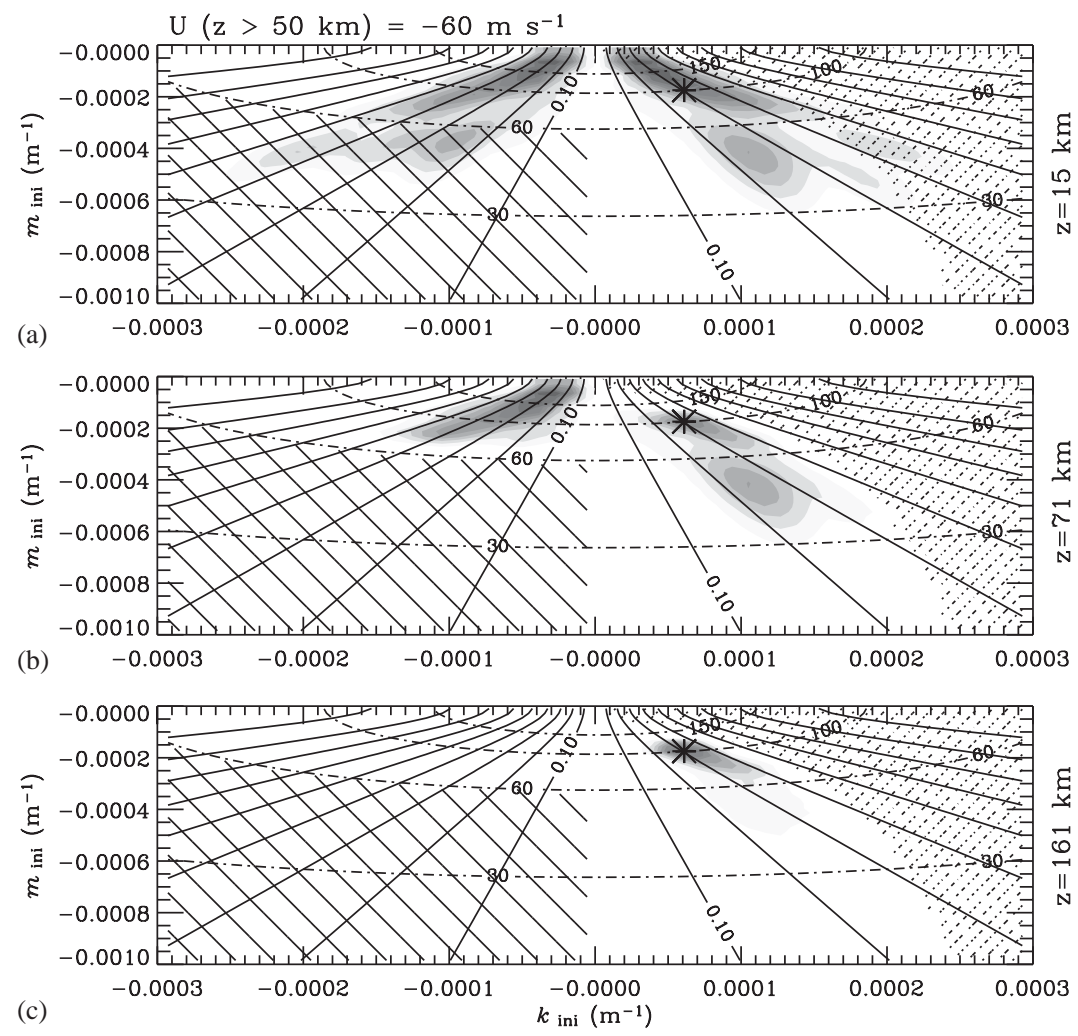

Fig. 17. Same as in Fig. 15, but for a background wind with $U_{0}=-60 \mathrm{~m} \mathrm{~s}^{-1}$. The asterisk show those waves with $\lambda_{x \text {,ini }}=103 \mathrm{~km}$ and $\lambda_{z, \text { ini }}=36 \mathrm{~km}$.

initiation of plume forcing (each representing a $\sim 30$-min average). The height, $z=161 \mathrm{~km}$, is the altitude of maximum deposited momentum. The thermospheric response is fairly simple, in that the spatial distribution is similar to a Gaussian, with total horizontal extent of $1000 \mathrm{~km}$. The response turns on smoothly at $\sim 1 \mathrm{~h}$, and lasts for a few hours, with the spatial distribution varying little during that time. Note that the gravity waves with $l \simeq 0$ contribute significantly to the thermospheric body forcings.

The main contributor to the total thermospheric response comes from the deepest plume, as can be seen in Figs. 12g $-\mathrm{i}$ at $z=161 \mathrm{~km}$. The response from the deepest plume has a similar spatial distribution as the total response. The total thermospheric response is localized in time, occurring for a few hours, which is a few times longer than the duration of the mesoscale convective complex. The thermospheric response to the deepest plume is approximately $30 \mathrm{~min}$, which is approximately twice the duration of this plume's duration. These are indications, then, that only certain waves with similar group velocities and frequencies escape filtering and dissipation at lower altitudes and deposit their momentum at these high altitudes. For, if other waves with significantly smaller group velocities were significantly involved at these altitudes, the thermospheric response would be distributed over a longer period of time. The observation that the pattern size and shape varies only slightly in time in Fig. 12 also indicates that the response at high altitudes depends less on the excited wave spectrum than on the characteristics of those waves which survive filtering and dissipation and penetrate to these high altitudes.

The full horizontal width of the deepest plume is $\simeq 18 \mathrm{~km}$, and the initial MCC area is $100 \mathrm{~km} \times 100 \mathrm{~km}$, as shown by the dotted box centered at $x=y=0$. Therefore, the thermospheric response to this deepest plume is spread horizontally by an order of magnitude, and is due to wave dispersion. This confirms the approximation we used for ray tracing, that the convective plume sources of gravity waves could be considered to be "point sources".

Finally, we consider the large momentum deposited in the thermosphere from this excitation mechanism. An average forcing in the thermosphere of $100 \mathrm{~m} \mathrm{~s}^{-1}$ day $^{-1}$ is equivalent to an average momentum flux of $8 \mathrm{~m}^{2} \mathrm{~s}^{-2}$ when spread over a scale height of $7 \mathrm{~km}$. Because the half-maximum of the total momentum fluxes shown in Fig. $12 \mathrm{~b}$ is $\simeq 100 \mathrm{~m}^{2} \mathrm{~s}^{-2}$, the ratio of the deposited momentum from this mechanism to the average momentum flux is $100 / 8 \sim 13$. This number may be somewhat lower if realistic wind profiles are used, as more filtering would likely occur, 
leading to a reduced gravity wave spectrum and smaller body forcings in the lower thermosphere. This ratio implies that the thermospheric forcings are likely much larger than the daily averages. If this mechanism is partly responsible for such a daily average, then thermospheric momentum flux divergence must occur intermittently in time and in space, as is expected from the nature of MCCs.

The corresponding vertical profile of the total, induced body force is shown as a function of time in Fig. 13a. Horizontal averaging was performed on the momentum fluxes prior to calculating the vertical derivative from Eq. (44). The thermospheric response is positive and is confined vertically, with no significant response below $150 \mathrm{~km}$ or above $170 \mathrm{~km}$ (with our assumption of thermospheric thermal structure). It is also confined temporally, with no significant response before $t=0.7 \mathrm{~h}$ or after $t=3.5 \mathrm{~h}$. The peak is maximum at $t=1.8 \mathrm{~h}$ and $z=163 \mathrm{~km}$. The pattern size and shape varies only slightly in time because the response at high altitudes depends less on the initial MCC wave spectrum than on those waves with the characteristics needed to survive filtering and dissipation and penetrate to high altitudes. Note, however, that the momentum flux divergence that occurs at later times (i.e., $t=2.5-3.5 \mathrm{~h}$ ) occurs at somewhat lower altitudes and is due to somewhat slower waves. Fig. $13 \mathrm{~b}$ shows the positive individual responses for the 5 deepest plumes. (The 5 deepest plumes are also the 5 largest plumes in terms of the total, integrated momentum flux; see Fig. 11b.) The maximum of each body force distribution occurs at $z \simeq 163-165 \mathrm{~km}$, which is a few kilometers above the maximum momentum deposition altitude. Again, the deepest plume contributes the most to the total deposited momentum. The times over which each of these plumes caused a body forcing varies mostly because of the varying excitation times. Subtracting the midpoint of the plume excitation time from the maximum forcing time for each of these 5 plumes, we find that the excited gravity waves take approximately $\simeq 1.2-1.3 \mathrm{~h}$ to propagate and dissipate in the thermosphere after their excitation. Because the shear is just above the plumes, this implies approximate vertical group velocities in the range $\sim 32-35 \mathrm{~m} \mathrm{~s}^{-1}$.

In order to understand the role that filtering, turning levels, and dissipation play on the survival of gravity waves in our model, we show several spectra as a function of altitude in Fig. 14 for the $l=0$ gravity waves only. The upper, middle, and lower panels show the gravity wave spectra at altitudes of $15 \mathrm{~km}$ (just above the excitation altitude for the MCC gravity waves), $71 \mathrm{~km}$ (just above the shear and resulting filtering effects), and at $161 \mathrm{~km}$ (at the maximum dissipation altitude), respectively. These gravity wave spectra include only those waves with $l=0$, which was motivated by Fig. 12 where we observed that large thermospheric responses occurred for waves propagating parallel to the background wind. In the top panel, we see that approximately equal amounts of westward and eastward traveling gravity waves are present, with the maxima occurring at $\lambda_{x} \sim 100 \mathrm{~km}$ and $\lambda_{z} \sim 30 \mathrm{~km}$ for these $l=0$ waves. The slight eastward-west- ward asymmetry is due to the fact that nearby multiple body forces acting at similar times create gravity wave fields that add and subtract coherently, producing an enhancement of positive over negative momentum flux, or vice versa (Vadas et al., 2003). Above the shear, waves with vertical wavelengths less than approximately $20 \mathrm{~km}$ are removed, and the spectral maximum shifts towards somewhat smaller horizontal wavelengths. At this altitude, the spectrum peaks at $\omega_{\text {Ir }} / N \sim 0.3$ to 0.5 and $\lambda_{x} \sim 40-250 \mathrm{~km}$. At the maximum dissipation level $(z=161 \mathrm{~km})$, the maximal vertical wavelengths are not very different than their values above the shear. Due to filtering effects and viscous dissipation, waves with horizontal wavelengths smaller than $45 \mathrm{~km}$ and larger than $250 \mathrm{~km}$, and waves with vertical wavelengths less than $\sim 25 \mathrm{~km}$ and greater than $65 \mathrm{~km}$, are no longer significant in the wave spectrum. The spectrum peaks at $\omega_{\text {Ir }} / N \sim 0.25$ -0.35 and $\lambda_{x} \sim 90-160 \mathrm{~km}$, implying intrinsic phase speeds of $\omega_{\text {Ir }} / k \sim 70-180 \mathrm{~m} \mathrm{~s}^{-1}$. Because these phase speeds are much larger than the horizontal velocity perturbations at $z=90 \mathrm{~km}$ depicted in Fig. 6, these waves are not expected to saturate below the turbopause. This confirms our assumption that saturation can be neglected for those waves most important to the forcing of the lower thermosphere at the highest altitudes.

In Fig. 15, we show the same gravity wave momentum flux spectrum as in Fig. 14, but as a function of the initial wave parameters $k_{\text {ini }}$ and $m_{\text {ini }}$ (i.e., at $z=13 \mathrm{~km}$ ). The gravity wave spectrum peaks at the highest altitude for waves with $\lambda_{x, \text { ini }}=103 \mathrm{~km}$ and $\lambda_{z \text {,ini }}=46 \mathrm{~km}$, yielding $\omega_{\text {ini }} / N \simeq 0.4$. This is marked by an asterisk in each of the three panels. This wave parameter occurs in the long-vertical-wavelength tail of the MCC distribution (denoted by an asterisk in Fig. 7). The cutoff in $\lambda_{z}$ for values of $80 \mathrm{~km}$ above the shear can be seen in this figure at small $m_{\mathrm{ini}}$. This cutoff may have lowered the peak vertical wavelength somewhat, although raising the cutoff vertical wavelength to $100 \mathrm{~km}$ has no significant effect on the thermospheric momentum flux and body force profiles. Only gravity waves with initial wavelengths of $\lambda_{x \text {,ini }} \simeq 50-200 \mathrm{~km}$ and $\lambda_{z \text {,ini }} \simeq 25-65 \mathrm{~km}$ contribute to the thermospheric body forcing at $z=161 \mathrm{~km}$ for this assumed shear, although larger vertical wavelengths (which cannot be studied here with our anelastic assumption) may be important as well. These results are, in general, a function of the shear and assumed MCC distribution.

We also display in Fig. 15 those waves that are removed from the gravity wave spectrum due to critical levels, which occurs when

$\omega_{\mathrm{Ir}}=\omega_{\mathrm{r}}-k U_{z>50 \mathrm{~km}} \leqslant 0$,

where $U_{z}>50 \mathrm{~km}$ is the mean, background, zonal wind above $50 \mathrm{~km}$ in altitude and equals $-30 \mathrm{~m} \mathrm{~s}^{-1}$ in Figs. 14 and 15 (and equals $-60 \mathrm{~m} \mathrm{~s}^{-1}$ in Figs. 16 and 17). We also display those waves that are excluded due to turning levels at lower altitudes within the shear layer, which occurs when

$m^{2}=\frac{k^{2} N^{2}}{\left(\omega_{\mathrm{r}}-k U_{z>50 \mathrm{~km}}\right)^{2}}-k^{2}-\frac{1}{4 H^{2}} \leqslant 0$. 
It is seen that critical level filtering does not significantly impact the westward propagating waves in Fig. 15, because the absolute value of the phase speeds are generally larger than $30 \mathrm{~m} \mathrm{~s}^{-1}$. It is also seen that filtering from evanescence within the shear layer does not significantly impact the eastward propagating waves because the background wind is not strong enough in this case (see Fig. 17 in comparison); turning levels within this shear only occur for those waves with very high frequencies, which are not well represented in our model MCC spectrum.

In order to understand how the evolving gravity wave spectrum with altitude depends on the background wind environment, we also ray trace the same $l=0$ gravity waves through a background zonal wind given by Eq. (43) with $U_{0}=-60 \mathrm{~m} \mathrm{~s}^{-1}$. The corresponding momentum fluxes are displayed in Figs. 16 and 17. Because the wind is much larger here, the eastward-propagating waves are refracted in the shear to larger vertical wavelengths than for the waves in Fig. 14. Therefore, it is no surprise that the surviving wave spectrum initially starts out at smaller frequencies, and that a good portion of the higher-frequency, eastward-propagating gravity waves are eliminated by evanescence and reflection within the stronger shear layer. Comparing Figs. 15c and $17 \mathrm{c}$, gravity waves with initially smaller vertical wavelengths and frequencies survive the shear and propagate to the highest altitudes for this stronger background wind. In this case, the gravity waves that are responsible for the peak body forcing at $z \simeq 161 \mathrm{~km}$ are waves with initial parameters of $\lambda_{x, \text { ini }} \simeq 103 \mathrm{~km}$ and $\lambda_{z \text {,ini }} \simeq 36 \mathrm{~km}$ (denoted by the triangle in Fig. 7). We also note that there is much more critical level filtering of the westward-propagating gravity waves, due to the fact that many of the westward-propagating waves have phase speeds that are smaller in absolute magnitude than $60 \mathrm{~m} \mathrm{~s}^{-1}$. It is important to note that the gravity wave spectrum that survives and dissipates at $z \simeq 161 \mathrm{~km}$ for both shears look very similar (compare Figs. 14c and 16c). This shows that viscous dissipation in the lower thermosphere preferentially acts to filter all $l=0$ waves except those with $\lambda_{x} \simeq 40-250 \mathrm{~km}$ and $\lambda_{z} \simeq 25-65 \mathrm{~km}$ at the highest altitudes, although waves with larger vertical wavelengths may also be important. The wave parameters and frequencies of the gravity waves within the initial MCC spectrum that have the biggest effect on the lower thermosphere, then, can be deduced by taking into account the filtering from viscous dissipation (Figs. 14c and 16c), and the refraction through intervening shears.

\subsection{Dissipation altitudes for realistic thermospheric temperatures}

The isothermal temperature profile $\bar{T}=250 \mathrm{~K}$ used in our ray-tracing simulations was for illustration and is much smaller than the actual thermospheric temperatures $(\bar{T} \geqslant 550 \mathrm{~K})$. The extreme minimum thermospheric temperature implies a density scale height of $\sim 14 \mathrm{~km}$, with even larger scale heights during solar maximum. Following
Pitteway and Hines (1963), the wave dissipation altitude depends on the local kinematic viscosity rather than on the viscosity profile. (See also Vadas and Fritts, 2004.) A larger density scale height implies that at a given altitude, the kinematic viscosity, $\mu / \bar{\rho}$, is smaller than it would be in an isothermal atmosphere. If we take a simple example of the temperature increasing from 250 to $500 \mathrm{~K}$ at an altitude of $125 \mathrm{~km}$, then the density decreases twice as slowly (and the kinematic viscosity increases twice as slowly) in altitude above $125 \mathrm{~km}$. Therefore, a gravity wave would propagate the same number of scale heights (thus twice as high) above $125 \mathrm{~km}$ for this simple example than for this isothermal temperature profile. We estimate that a wave that dissipates at $163 \mathrm{~km}$ in this isothermal atmosphere would instead dissipate at $125+2(163-$ $125) \simeq 201 \mathrm{~km}$ with this simple step-function temperature profile.

We obtain a better estimate by considering a realistic temperature profile. Fig. 18a shows an extreme minimum thermospheric temperature profile. Using $\mathrm{d} \bar{p} / \mathrm{d} z=-g \bar{\rho}$ and $\bar{p}=R \bar{\rho} \bar{T}$,

$\bar{p}=p_{\mathrm{s}} \exp \left(-\frac{1}{R} \int_{0}^{z} \frac{g}{\bar{T}} \mathrm{~d} z\right)$,

$\bar{\rho}=\bar{p} /(R \bar{T}), \mathrm{H}=-\bar{\rho} /(\mathrm{d} \bar{\rho} / \mathrm{d} z)$, and $N^{2}=(g / \bar{\theta}) \mathrm{d} \bar{\theta} / \mathrm{d} z$. The solid line in Fig. $18 \mathrm{~b}$ shows the altitude at which $\bar{\rho}$ for this extreme minimum temperature profile is equivalent to $\bar{\rho}$ for this isothermal profile. The density (and therefore the kinematic viscosity) at $165 \mathrm{~km}$ in the isothermal profile is equivalent to that at $\sim 200 \mathrm{~km}$ in the extreme minimum temperature profile. In addition, the increase in temperature also leads to a decrease in the buoyancy frequency, and therefore to larger vertical wavelengths and group velocities which likely result in somewhat higher penetration altitudes. Thus, we expect that our simulations underestimate the altitudes to which MCC gravity waves penetrate under realistic thermospheric temperature profiles. We anticipate that the thermospheric body forcings which result from the MCC gravity waves will likely be at altitudes of $z \sim 200 \mathrm{~km}$ under extreme minimum temperature conditions, and higher during solar maximum.

\subsection{Potential for ESF seeding}

Strong equatorial spread F (ESF) is a manifestation of a Rayleigh-Taylor instability (RTI) of the F region of the ionosphere (or alternatively, positively buoyant plasma bubbles) that may penetrate to $\sim 1000 \mathrm{~km}$ altitude, exhibits variability on $\sim$ daily to solar-cycle time scales and is believed by some researchers to be triggered by gravity waves. Three conditions appear to be required for gravity waves to participate effectively in the seeding of ESF. These are (1) wave penetration to altitudes at which seeding can occur, (2) sufficient wave amplitudes at seeding altitudes to trigger RTI and ESF on time scales consistent with observed events, and (3) phase speeds that can yield the resonance condi- 

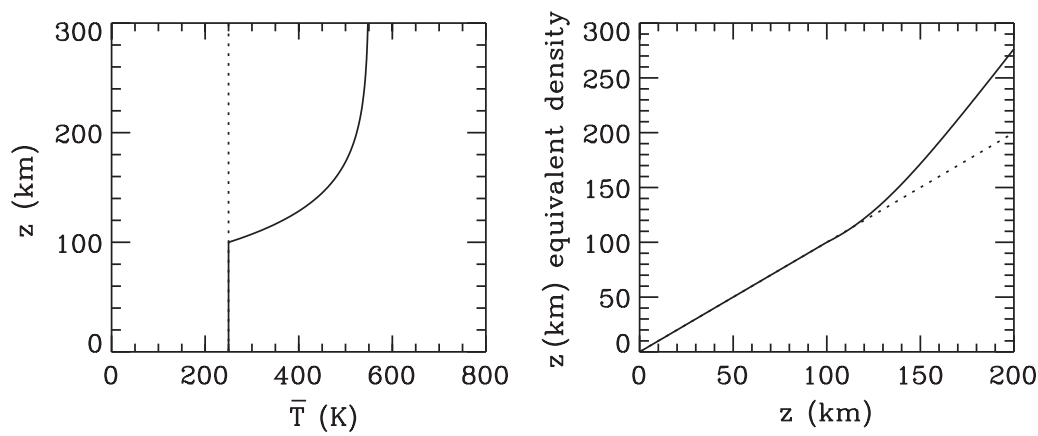

Fig. 18. (a) Extreme minimum thermospheric temperature profile (solid line). Dotted line is the isothermal temperature profile used for the simulations in this paper. (b) The altitude of equivalent density for the temperature profiles in (a) as compared to the isothermal temperature profile. The solid line shows the altitude of the equivalent density of the extreme minimum temperature profile as compared to the isothermal temperature profile.

tions suggested for successful coupling of wave and plasma processes (Hysell et al., 1990; Huang and Kelley, 1996a, b). The first of these requirements appears to be satisfied with waves penetrating to altitudes of $\sim 200 \mathrm{~km}$ at the magnetic equator or lower altitudes for magnetic latitudes up to $\sim \pm 20^{\circ}$ (estimating the effect of increased thermospheric temperatures on the dissipation altitude; see Section 5.3). The second condition is more challenging to assess from ray-tracing results alone, as the cumulative momentum flux for a single plume may be significant, but it is not yet clear how much of the wave energy (and amplitude) accounting for this flux is confined to a single region. Much of the momentum flux and wave activity for a deep, single plume is quite concentrated spatially and temporally (see Figs. $12 \mathrm{~g}-$ i) with amplitudes that are likely sufficiently large. Finally, gravity wave intrinsic phase speeds are correlated with the vertical wave scales at any altitude. These need to couple to horizontal plasma motions which move relative to the neutral fluid at these altitudes. Nevertheless, there is again sufficient variability in the gravity wave phase speeds and in the relative plasma-neutral motions, with comparable magnitudes, to suggest that such resonance conditions will occur in response to MCC gravity waves.

\section{Summary and conclusions}

We have employed two models to examine the excitation of gravity waves by MCC convection at equatorial latitudes and their propagation to, and possible influences in, the mesosphere, thermosphere, and ionosphere. A linear model of the responses to local body forces was employed to represent convective plumes and the spatial, temporal, and spectral character of the gravity waves excited by them. A ray-tracing model was then employed to propagate gravity waves comprising the spectrum through a mean wind shear and into an increasingly dissipative thermosphere.
The body force model specified convective plumes on spatial and temporal scales consistent with such plumes described in explicit models of tropical convection (Piani et al., 2000; Lane et al., 2001). The model was first used to assess the effects of single and multiple plumes and plume spacing on the scales and frequencies of gravity waves excited in this manner. Results for a single plume revealed a tendency for vertical motions to excite gravity waves having higher frequencies and smaller horizontal scales than a horizontal body forcing having the same geometry. High-frequency gravity waves are preferentially excited by deep, strong plumes of limited horizontal extent.

Results for multiple plumes revealed that closely spaced coherent plumes (with separations less than $\sim 2$ plume diameters) lead to enhanced excitation of larger horizontal scales than arise from a single plume. Plume spacings of $\sim 2$ plume diameters or larger, however, yield only a weak enhancement of waves at larger horizontal scales and a dominant peak near that for a single plume. This fact was used to justify describing the field of gravity waves arising from a collection of convective plumes as the superposition of the wave fields arising from each plume individually, provided the spacings between strong and deep plumes are sufficiently large.

Ray tracing of components of the gravity wave spectrum arising from convective plumes through a mean wind shear reveals that penetration to high altitudes is preferred for waves refracting to (1) high intrinsic frequencies of order $\omega_{\text {Ir }} / N \sim 0.3-0.6$, and (2) large vertical wavelengths of order $\lambda_{z} \sim 25-65 \mathrm{~km}$. Gravity waves with even larger vertical wavelengths may also be important, although we were unable to simulate them due to limitations of our Boussinesq body force model and anelastic ray-trace model. These waves dissipated at $\simeq 150-170 \mathrm{~km}$ (with our assumption of an isothermal thermal structure). Due to wave dispersion, the thermospheric response was spread spatially by an order of magnitude in each horizontal direction from the MCC, leading to instantaneous forcings of order $(1000 \mathrm{~km})^{2} \times 25 \mathrm{~km}$. 
The thermospheric response was found to occur from $\sim 0.7$ $-3.5 \mathrm{~h}$ after initiation of convective excitation, with individual responses from plumes lasting approximately $30 \mathrm{~min}$. The largest thermospheric responses were found to occur for the deepest plumes. Variable winds were found to impose significant anisotropy on the surviving gravity waves, causing large net momentum fluxes and flux divergence well into the thermosphere.

Increasing the background wind at lower altitudes had the effect of filtering those waves with higher frequencies and group velocities via evanescence and reflection within the shear layer. This increased refraction had the effect of greatly increasing the frequency and group velocity of those waves with initially smaller frequencies and group velocities. Thus, the portion of the MCC gravity wave spectrum that survived the winds and dissipated at the highest altitudes depended on the background wind environment through which it passed in our model studies. We also found that for background winds of 30 and $60 \mathrm{~m} \mathrm{~s}^{-1}$, viscous dissipation in the lower thermosphere preferentially acted to filter out all waves but those with $\lambda_{x} \simeq 40-250 \mathrm{~km}$ and $\lambda_{z} \simeq 25-65 \mathrm{~km}$ at the highest altitudes. Larger vertical wavelength waves may also be important, although we are limited to gravity waves with $\lambda_{z}<4 \pi H \simeq 88 \mathrm{~km}$ in this study.

These results were obtained assuming an isothermal temperature structure throughout the atmosphere of $\bar{T}=250 \mathrm{~K}$. However, the lower thermospheric temperature is at least $\bar{T}=500 \mathrm{~K}$, depending on solar conditions. This results in a gravity wave penetration altitude that is significantly higher than that calculated here (Vadas and Fritts, 2004), and is estimated to be $\sim 200 \mathrm{~km}$ under extreme minimum temperature conditions, and higher during solar maximum.

Gravity waves excited by MCCs and having sufficiently large vertical wavelengths and group velocities above the shears at lower altitudes can thus penetrate to altitudes at which seeding of RTI and ESF is believed to occur when we include larger thermospheric temperature effects. Our results further suggest that wave amplitudes may be sufficiently large for such seeding and that, at least on occasion, wave phase speeds and plasma drifts are sufficiently aligned to satisfy the spatial resonance conditions believed to be required for the seeding of ESF.

\section{Acknowledgements}

This research was supported by the National Aeronautics and Space Administration under contracts NAS5-02036 and NAS5-01075, the National Science Foundation under grant ATM-0307910, and the Air Force Office of Scientific Research under contracts F49620-00-C-0008 and F49620-03-C-0045. DCF also thanks ESRANGE, Kiruna, Sweden, for warm hospitality during the preparation of this paper.

\section{References}

Alexander, M.J., Holton, J.R., 1997. A model study in the equatorial stratosphere by convectively induced gravity waves. Journal of Atmospheric Science 54, 408-419.

Alexander, M.J., Pfister, L., 1995. Gravity wave momentum flux in the lower stratosphere over convection. Geophysical Research Letters 22, 2029-2032.

Alexander, M.J., Vincent, R.A., 2000. Gravity waves in the tropical lower stratosphere: a model study of seasonal and interannual variability. Journal of Geophysical Research 105, 17983-17993.

Alexander, M.J., Holton, J.R., Durran, D.R., 1995. The gravity wave response above deep convection in a squall line simulation. Journal of Atmospheric Science 52, 2212-2226.

Alexander, M.J., Beres, J.H., Pfister, L., 2000. Tropical stratospheric gravity wave activity and relationships to clouds. Journal of Geophysical Research 105, 22299-22309.

Beres, J.H., Alexander, M.J., Holton, J.R., 2002. Effects of tropospheric wind shear on the spectrum of convectively generated gravity waves. Journal of Atmospheric Science 59, 1805-1824.

Beres, J.H., Alexander, M.J., Holton, J.R., 2004. A method of specifying the gravity wave spectrum above convection based on latent heating properties and background wind. Journal of Atmospheric Science 61, 324-336.

Chun, H.Y., Baik, J.J., 1998. Momentum flux by thermally induced internal gravity waves and its approximation by large-scale models. Journal of Atmospheric Science 55, 3299-3310.

Clark, T.E., Hauf, T., Kuettner, J.P., 1986. Convectively forced internal gravity waves: results from two-dimensional numerical experiments. Quarterly Journal of Royal Meteorological Society 112, 899-925.

Dewan, E.M., Picard, R.H., ONeil, R.R., Gardiner, H.A., Gibson, J., Mill, J.D., Richards, E., Kendra, M., Gallery, W.O., 1998. MSX satellite observations of thunderstorm-generated gravity waves in mid-wave infrared images of the upper stratosphere. Geophysical Research Letters 25, 939-942.

Dhaka, S.K., Devrajan, P.K., Shibagaki, Y., Choudhary, R.K., Fukao, S., 2001. Indian MST radar observations of gravity wave activities associated with tropical convection. Journal of Atmospheric and Solar Terrestrial Physics 63, 1631-1642.

Dhaka, S.K., Choudhary, R.K., Malik, S., Shibagaki, Y., Yamanaka, M.D., Fukao, S., 2002. Observable signatures of a convectively generated wave field over the tropics using Indian MST radar at Gadanki $\left(13.5^{\circ} \mathrm{N}, 79.2^{\circ} \mathrm{E}\right)$. Geophysical Research Letters 29, 10.1029/2002GL014745.

Dunkerton, T.J., 1997. The role of gravity waves in the quasi-biennial oscillation. Journal of Geophysical Research 102, 26053-26076.

Eckermann, S.D., 1992. Ray-tracing simulation of the global propagation of inertia gravity waves through the zonally-averaged middle atmosphere. Journal of Geophysical Research 97, 15849-15866.

Eckermann, S.D., Marks, C.J., 1996. An idealized ray model of gravity wave-tidal interactions. Journal of Geophysical Research 101, 21195-21212.

Fovell, R., Durran, D., Holton, J.R., 1992. Numerical simulation of convectively generated gravity waves. Journal of Atmospheric Science 49, 1427-1442. 
Fritts, D.C., Alexander, M.J., 2003. Gravity wave dynamics and effects in the middle atmosphere. Reviews on Geophysics 41, 10.1029/2001RG000106.

Fritts, D.C., Luo, Z., 1992. Gravity wave excitation by geostrophic adjustment of the jet stream, Part 1: two-dimensional forcing. Journal of Atmospheric Science 49, 681-697.

Fritts, D.C., Nastrom, G.D., 1992. Sources of mesoscale variability of gravity waves, II: frontal, convective, and jet stream excitation. Journal of Atmospheric Science 49, 111-127.

Hauf, T., 1993. Aircraft observation of convection waves over southern Germany - a case study. Monthly Weather Review 121, 3282-3290.

Hocke, K., Schlegel, K., 1996. A review of atmospheric gravity waves and traveling ionospheric disturbances: 1982-1995. Annales of Geophysics 14, 917-940.

Hocke, K., Tsuda, T., 2001. Gravity waves and ionospheric irregularities over tropical convection zones observed by GPS/MET radio occultation. Geophysical Research Letters 28, 2815-2818.

Holton, J.R., Beres, J.H., Zhou, X., 2002. On the vertical scale of gravity waves excited by localized thermal forcing. Journal of Atmospheric Science 59, 2019-2023.

Horinouchi, T., Nakamura, T., Kosaka, J., 2002. Convectively generated mesoscale gravity waves simulated throughout the middle atmosphere. Geophysical Research Letters 29, 10.1029/2002GL016069.

Huang, C.S., Kelley, M.C., 1996a. Nonlinear evolution of equatorial spread F, 2, gravity wave seeding of Rayleigh-Taylor instability. Journal of Geophysical Research 101, 293.

Huang, C.S., Kelley, M.C., 1996b. Nonlinear evolution of equatorial spread F, 4, gravity waves, velocity shear, and day-to-day variability. Journal of Geophysical Research 101, 24523.

Hysell, D.L., Kelley, M.C., Swartz, W.E., Woodman, R.F., 1990. Seeding and layering of equatorial spread F. Journal of Geophysical Research 95, 17253.

Karoly, D.J., Roff, G.L., Reeder, M.J., 1996. Gravity wave activity associated with tropical convection detected in TOGA COARE sounding data. Geophysical Research Letters 23, 261-264.

Kuettner, J.P., Hildebrand, P.A., Clark, T.L., 1987. Convection waves: observation of gravity wave systems over convectively active boundary layers. Quarterly Journal of Royal Meteorological Society 113, 445-467.

Kundu, P., 1990. Fluid Dynamics. Academic Press, San Diego, pp. 638.

Lane, T.P., Reeder, M.J., Clark, T.L., 2001. Numerical modeling of gravity waves generated by deep tropical convection. Journal of Atmospheric Science 58, 1249-1274.

Larsen, M.F., Swartz, W.E., Woodman, R.F., 1982. Gravity-wave generation by thunderstorms observed with a vertically-pointing $430 \mathrm{MHz}$ radar. Geophysical Research Letters 9, $571-574$.

Lighthill, J., 1978. Waves in Fluids. Cambridge University Press, Cambridge, pp. 504.

Lu, D., VanZandt, T.E., Clark, W.L., 1984. VHF Doppler radar observations of buoyancy waves associated with thunderstorms. Journal of Atmospheric Science 41, 272-282.

Luo, Z., Fritts, D.C., 1993. Gravity wave excitation by geostrophic adjustment of the jet stream, Part II: three-dimensional forcing. Journal of Atmospheric Science 50, 104-115.
Marks, C.J., Eckermann, S.D., 1995. A three-dimensional nonhydrostatic ray-tracing model for gravity waves: formulation and preliminary results for the middle atmosphere. Journal of Atmospheric Science 52, 1959-1984.

Oliver, W.L., Otsuka, Y., Sato, M., Takami, T., Fukao, S., 1997. A climatology of $\mathrm{F}$ region gravity wave propagation over the middle and upper atmosphere radar. Journal of Geophysical Research 102, 14499-14512.

Pandya, R.E., Alexander, M.J., 1999. Linear stratospheric gravity waves above convective thermal forcing. Journal of Atmospheric Science 56, 2434-2446.

Pfister, L., Chan, K.R., Bui, T.P., Bowen, S., Legg, M., Gary, B., Kelly, K., Proffitt, M., Starr, W., 1993a. Gravity waves generated by a tropical cyclone during the STEP tropical field program: a case study. Journal of Geophysical Research 98, 8611-8638.

Pfister, L., Scott, S., Loewenstein, M., Bowen, S., Legg, M., 1993 b. Mesoscale disturbances in the tropical stratosphere excited by convection: observations and effects on the stratospheric momentum budget. Journal of Atmospheric Science 50, $1058-1075$.

Piani, C., Durran, D., Alexander, M.J., Holton, J.R., 2000. A numerical study of three dimensional gravity waves triggered by deep tropical convection. Journal of Atmospheric Science 57, 3689-3702.

Pitteway, M.L.V., Hines, C.O., 1963. The viscous damping of atmospheric gravity waves. Canadian Journal of Physics 41, 1935-1948.

Press, W.H., Teukolsky, S.A., Vetterling, W.T., Flannery, B.P., 1992. Numerical Recipes in Fortran, 2nd Edition. Cambridge University Press, Cambridge, 963pp.

Salby, M.L., Garcia, R.R., 1987. Transient response to localized episodic heating in the tropics, Part I: excitation and short-time near-field behavior. Journal of Atmospheric Science 44, $458-498$.

Sato, K., Hashiguchi, H., Fukao, S., 1995. Gravity waves and turbulence associated with cumulus convection observed with the UHF/VHF clear-air Doppler radars. Journal of Geophysical Research 100, 7111-7119.

Sekar, R., Kelley, M.C., 1998. On the combined effects of vertical shear and zonal electric field patterns on nonlinear equatorial spread F evolution. Journal of Geophysical Research 103, 20735-20747.

Sentman, D.D., Wescott, E.M., Picard, R.H., Winick, J.R., Stenbaek-Nielsen, H.C., Dewan, E.M., Moudry, D.R., Sao Sabbas, F.T., Heavner, M.J., Morrill, J., 2003. Simultaneous observations of mesospheric gravity waves and sprites generated by a midwestern thunderstorm. Journal of Atmospheric and Solar Terrestrial Physics 65, 5.

Taylor, M.J., Hapgood, M.A., 1988. Identification of a thunderstorm as a source of short period gravity waves in the upper atmospheric nightglow emissions, Planetary and Space Science 36, 975.

Tsuda, T., Nishida, M., Rocken, C., Ware, R.H., 2000. A global morphology of gravity wave activity in the stratosphere revealed by the GPS occultation data (GPS/MET). Journal of Geophysical Research 105, 7257-7273.

Vadas, S.L., Fritts, D.C., 2001. Gravity wave radiation and mean responses to local body forces in the atmosphere. Journal of Atmospheric Science 58, 2249-2279.

Vadas, S.L., Fritts, D.C., 2002. The importance of spatial variability in the generation of secondary gravity waves 
from local body forces. Geophysical Research Letters 29, 10.1029/2002GL015574.

Vadas, S.L., Fritts, D.C., 2004. Thermospheric responses to gravity waves: influences of increasing viscosity and thermal diffusivity. In preparation.

Vadas, S.L., Fritts, D.C., Alexander, M.J., 2003. Mechanism for the generation of secondary waves in wave breaking regions. Journal of Atmospheric Science 60, 194-214.
Vincent, R.A., Alexander, M.J., 2000. Gravity waves in the tropical lower stratosphere: an observational study of seasonal and interannual behavior. Journal of Geophysical Research 105, 17971-17982.

Walterscheid, R.L., Schubert, G., Brinkman, D.G., 2001. Small-scale gravity waves in the upper mesosphere and lower thermosphere generated by deep tropical convection. Journal of Geophysical Research 106, 31825-31832. 